\title{
The Conformational Landscape of $m$-Anisic Acid and its Complexes with Formic Acid
}

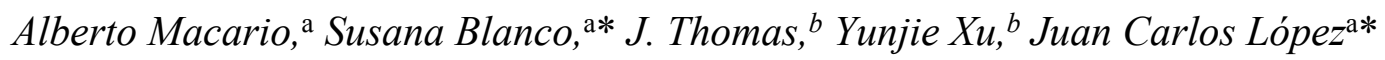

a Dpto. Química Física y Química Inorgánica, Facultad de Ciencias, IU Cinquima, Universidad de Valladolid, Paseo Belén 7, 47011-Valladolid, SPAIN.

${ }^{\mathrm{b}}$ Department of Chemistry, University of Alberta, Edmonton, Alberta, CANADA, T6G 2G2 


\section{Supporting Information}

\section{Contents:}

Complete reference 34

Figure S1. Predicted stable conformers of $m$-anisic acid monomer, and the observed conformers of $o$-anisic acid [Ref 25].

Figure S2. Predicted stable conformers of $m$-anisic acid - formic acid heterodimer, and the observed conformers of $o$-anisic acid - formic acid [Ref 21].

Figure S3. Comparison of the CP-FTMW spectrum of $m$-anisic acid and $m$-anisic acid-formic acid complex.

Figure S4. Potential energy functions predicted at MP2/aug-cc-pVDZ level of theory for the interconversion pathway between $\mathrm{A} 1$ - A2 and B1 - B2 forms of $m$-anisic acid monomer.

Figure S5. Potential energy functions predicted at MP2/aug-cc-pVDZ level of theory for the interconversion pathway between A1 - B1 and A2 - B2 forms of $m$-anisic acid monomer.

Figure S6. Potential energy functions predicted at MP2/aug-cc-pVDZ level of theory for the interconversion pathway between A1-F1 - A2-F1 and B1-F1 - B2-F1 forms of $m$-anisic acid - formic acid heterodimer.

Figure S7. Potential energy functions predicted at MP2/aug-cc-pVDZ level of theory for the interconversion pathway between A1-F1 - B1-F1 and A2-F1 - B2-F1 forms of $m$-anisic acid - formic acid heterodimer.

Table S1. Rotational parameters predicted from B3LYP-D3/6-311++G(d,p) level of theory for the stable conformers of $m$-anisic acid - formic acid heterodimer.

Table S2. Predicted $r_{\mathrm{e}}$ geometry parameters calculated at B3LYP-D3/6-311++G(d,p) level of theory for the four observed conformers of $m$-anisic acid monomer.

Table S3. Predicted $r_{\text {e }}$ geometry parameters calculated at B3LYP-D3/6-311++G(d,p) level of theory for the four observed conformers of $m$-anisic acid - formic acid heterodimer.

Table S4-S11. Transition frequencies of all the observed species of $m$-anisic acid and $m$ anisic acid - formic acid heterodimer. 
Complete reference 34:

Gaussian 09, Revision D.01, Frisch, M. J.; Trucks, G. W.; Schlegel, H. B.; Scuseria, G. E.; Robb, M. A.; Cheeseman, J. R.; Scalmani, G.; Barone, V.; Petersson, G. A.; Nakatsuji, H.; Li, X.; Caricato, M.; Marenich, A.; Bloino, J.; Janesko, B. G.; Gomperts, R.; Mennucci, B.; Hratchian, H. P.; Ortiz, J. V.; Izmaylov, A. F.; Sonnenberg, J. L.; Williams-Young, D.; Ding, F.; Lipparini, F.; Egidi, F.; Goings, J.; Peng, B.; Petrone, A.; Henderson, T.; Ranasinghe, D.; Zakrzewski, V. G.; Gao, J.; Rega, N.; Zheng, G.; Liang, W.; Hada, M.; Ehara, M.; Toyota, K.; Fukuda, R.; Hasegawa, J.; Ishida, M.; Nakajima, T.; Honda, Y.; Kitao, O.; Nakai, H.; Vreven, T.; Throssell, K.; Montgomery Jr., J. A.; Peralta, J. E.; Ogliaro, F.; Bearpark, M.; Heyd, J. J.; Brothers, E.; Kudin, K. N.; Staroverov, V. N.; Keith, T.; Kobayashi, R.; Normand, J.; Raghavachari, K.. Rendell, A.; Burant, J. C.; Iyengar, S. S.; Tomasi, J.; Cossi, M.; Millam, J. M.; Klene, M.; Adamo, C.; Cammi, R.; Ochterski, J. W.; Martin, R. L.; Morokuma, K.; Farkas, O.; Foresman, J. B.; Fox, D. J. Gaussian 09, Revision D.01, Gaussian, Inc., Wallingford CT, 2016. 
Figure S1. a) Stable conformers predicted for $m$-anisic acid monomer. Their corresponding parameters are given in Table 1. b) Observed conformers of $o$-anisic acid monomer [Ref 25]. The energies, relative to the lower energy conformer, are calculated at B3LYP-D3/6-311++G(d,p).

a)

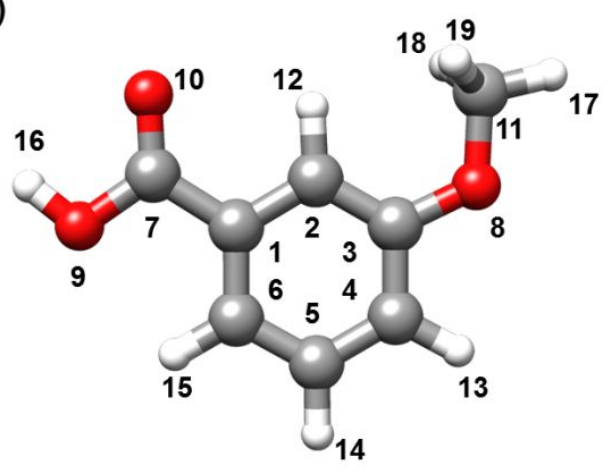

A1

$0.0 \mathrm{~cm}^{-1}$

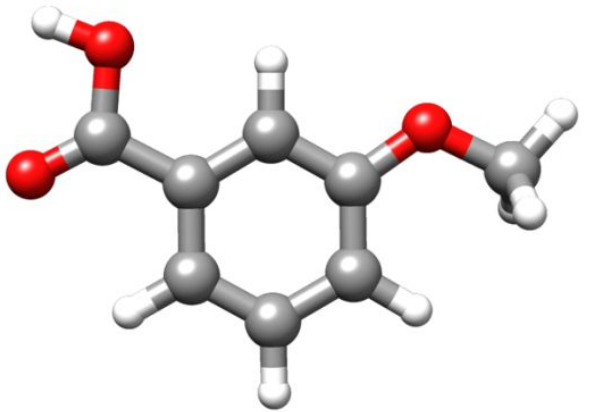

B2

$274.3 \mathrm{~cm}^{-1}$

b)

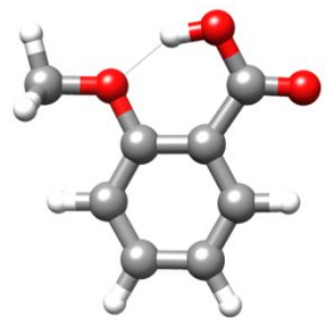

T1

$0.0 \mathrm{~cm}^{-1}$

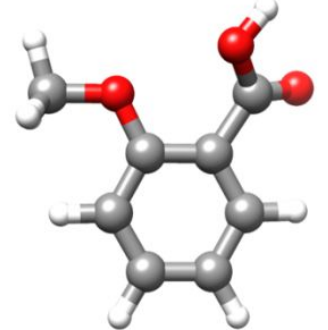

C2

$768.6 \mathrm{~cm}^{-1}$

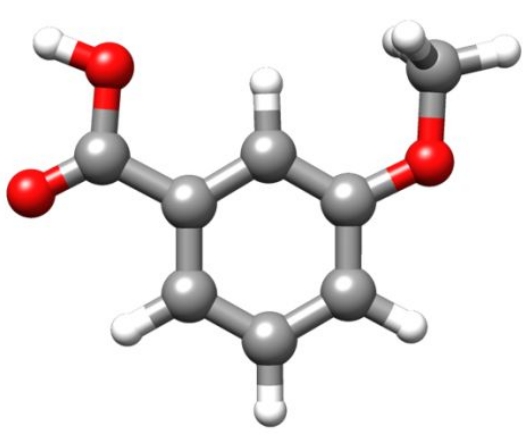

A2

$121.7 \mathrm{~cm}^{-1}$

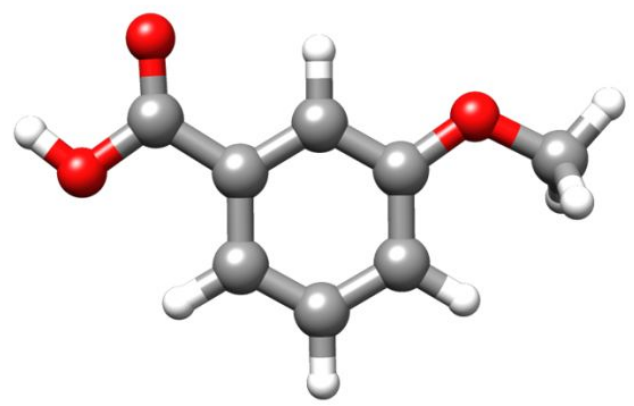

B1

$305.8 \mathrm{~cm}^{-1}$ 
Figure S2. a) Stable conformers predicted for $m$-anisic acid - formic acid heterodimer. Their corresponding parameters are given in Table S1. b) Observed conformers of $o$-anisic acid - formic acid heterodimer [Ref 21]. The energies, relative to the lower energy conformer, are calculated at B3LYP$\mathrm{D} 3 / 6-311++\mathrm{G}(\mathrm{d}, \mathrm{p})$.

a)
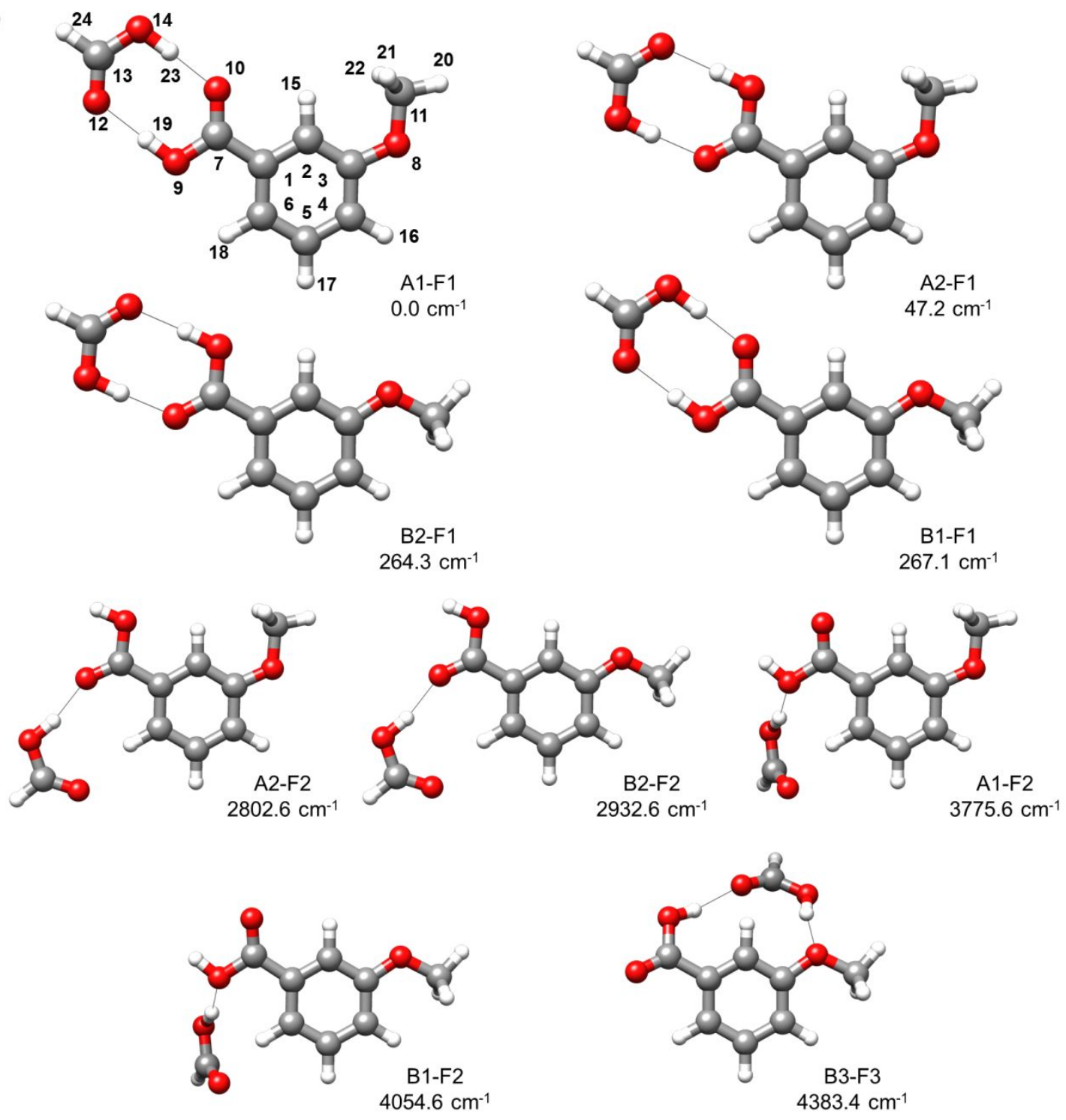

b)
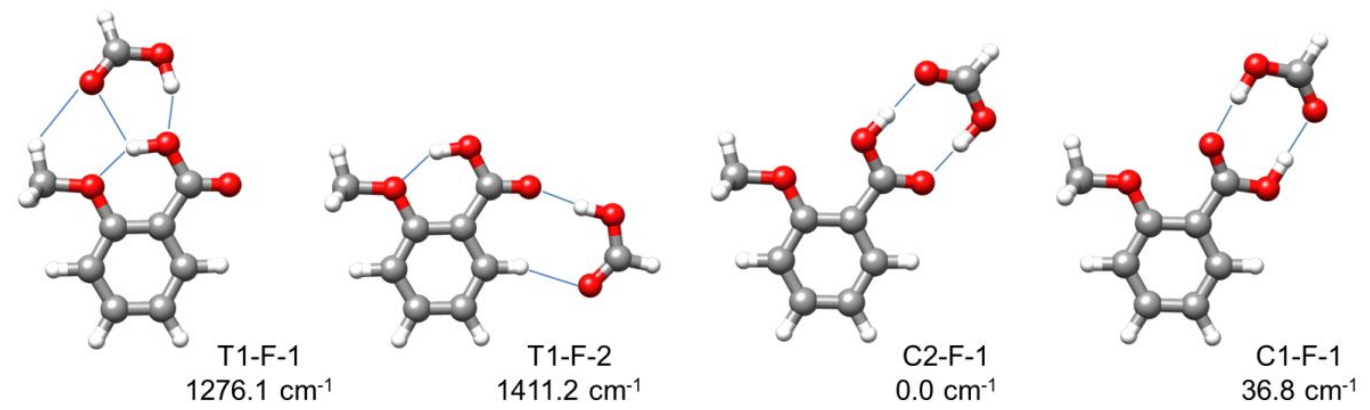
Figure S3. Comparison of the CP-FTMW spectrum of $m$-anisic acid (upper trace) and $m$-anisic acidformic acid complex (lower trace), together with the predicted spectra for each observed conformer. In the heterodimer spectrum all lines of $m$-anisic acid, formic acid and all other known species have been removed.

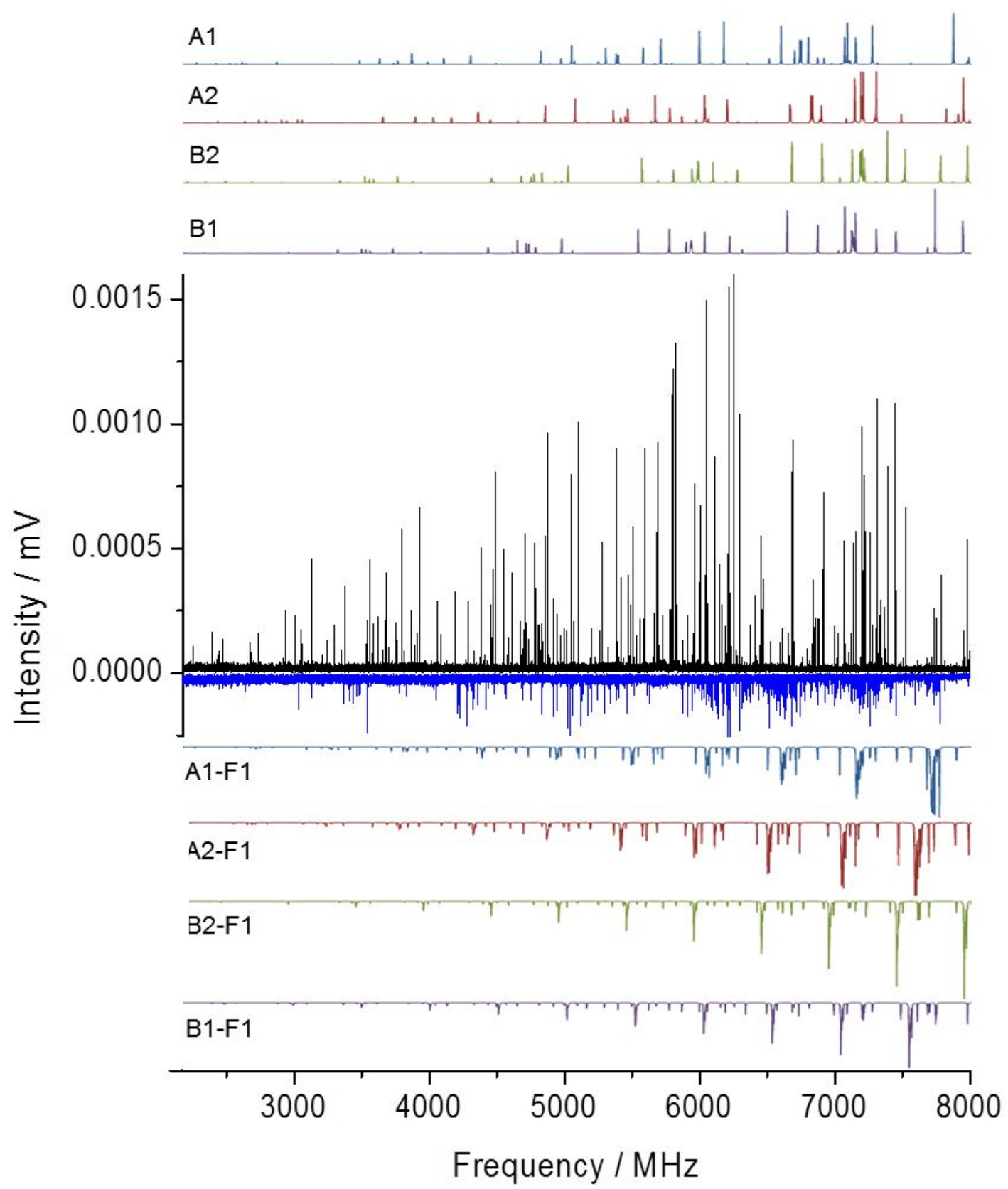


Figure S4. Potential energy functions predicted at MP2/aug-cc-pVDZ level of theory for the interconversion pathway between $\mathrm{A} 1$ - $\mathrm{A} 2$ and $\mathrm{B} 1$ - B2 forms of $m$-anisic acid monomer through the dihedral angle dih9 $=\mathrm{C}_{2}-\mathrm{C}_{1}-\mathrm{C}_{7}-\mathrm{O}_{9}$ (See Figure $\mathrm{S} 1$ for atom labelling).
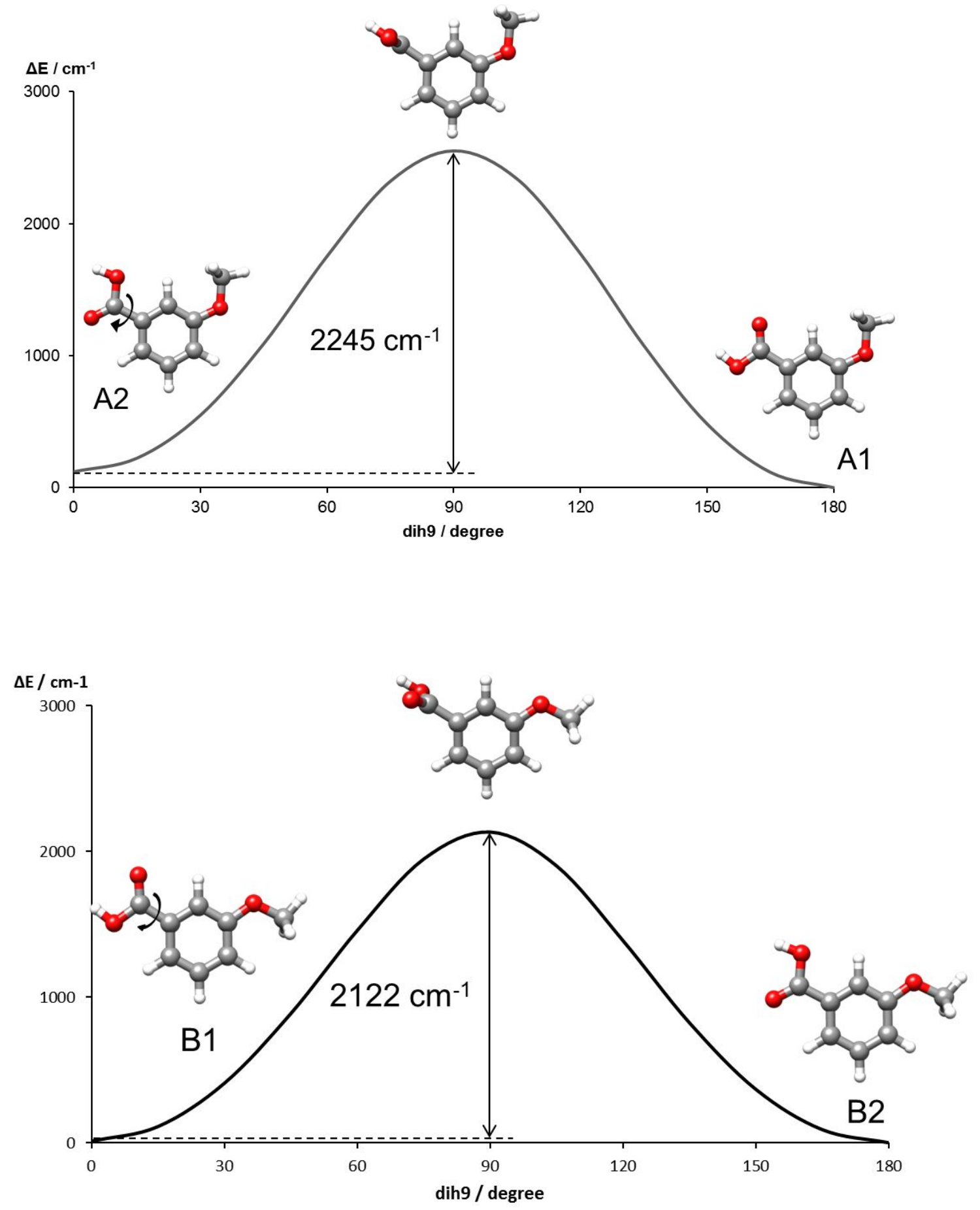
Figure S5. Potential energy functions predicted at MP2/aug-cc-pVDZ level of theory for the interconversion pathway between $\mathrm{A} 1$ - B1 and A2 - B2 forms of $m$-anisic acid monomer through the dihedral angle dih11 $=\mathrm{C}_{2}-\mathrm{C}_{3}-\mathrm{O}_{8}-\mathrm{C}_{11}$ (See Figure $\mathrm{S} 1$ for atom labelling).
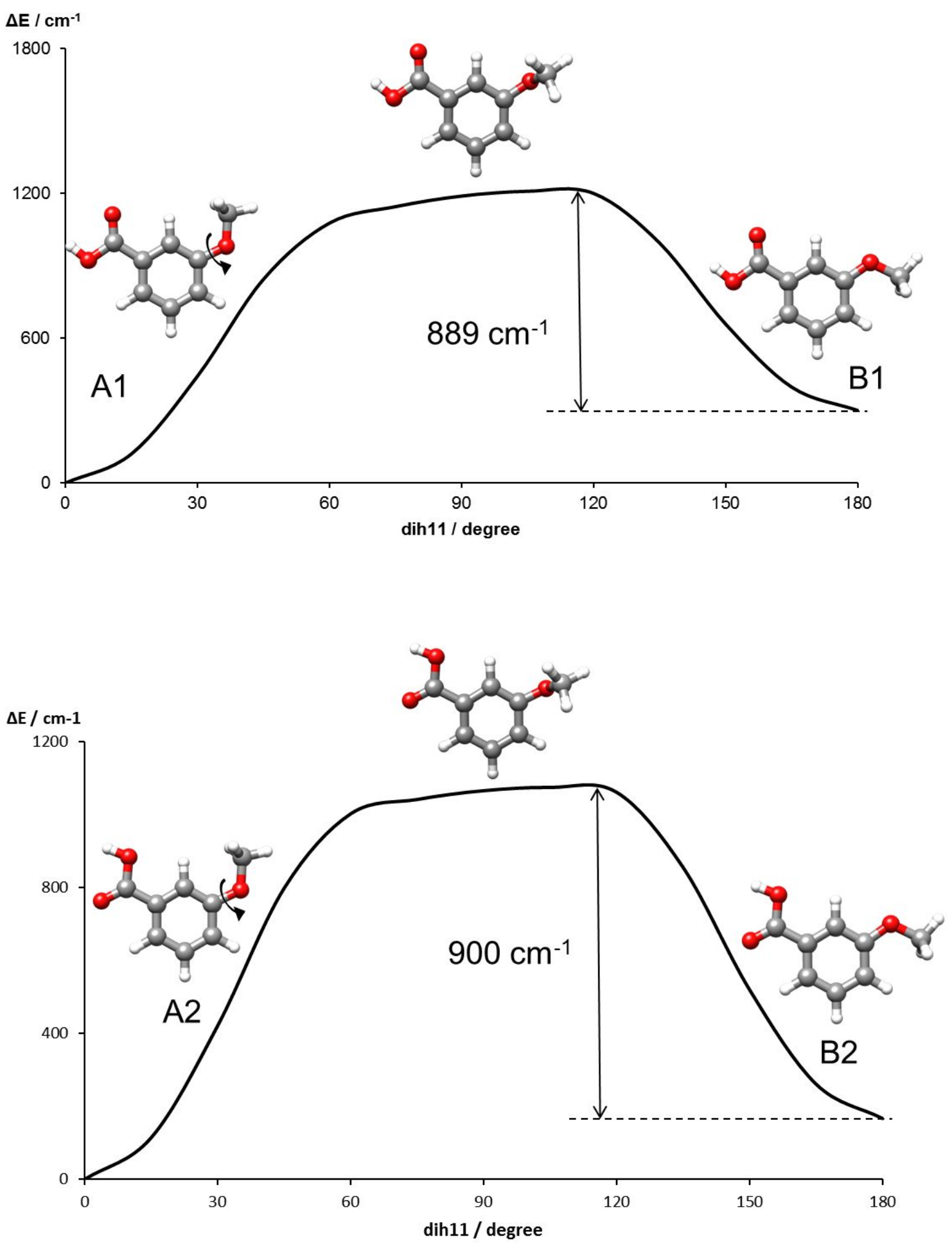
Figure S6. Potential energy functions predicted at MP2/aug-cc-pVDZ level of theory for the interconversion pathway between A1-F1 - A2-F1 and B1-F1 - B2-F1 forms of $m$-anisic acid - formic acid heterodimer through the dihedral angle dih $9=\mathrm{C}_{2}-\mathrm{C}_{1}-\mathrm{C}_{7}-\mathrm{O}_{9}$ (See Figure S2 for atom labelling).
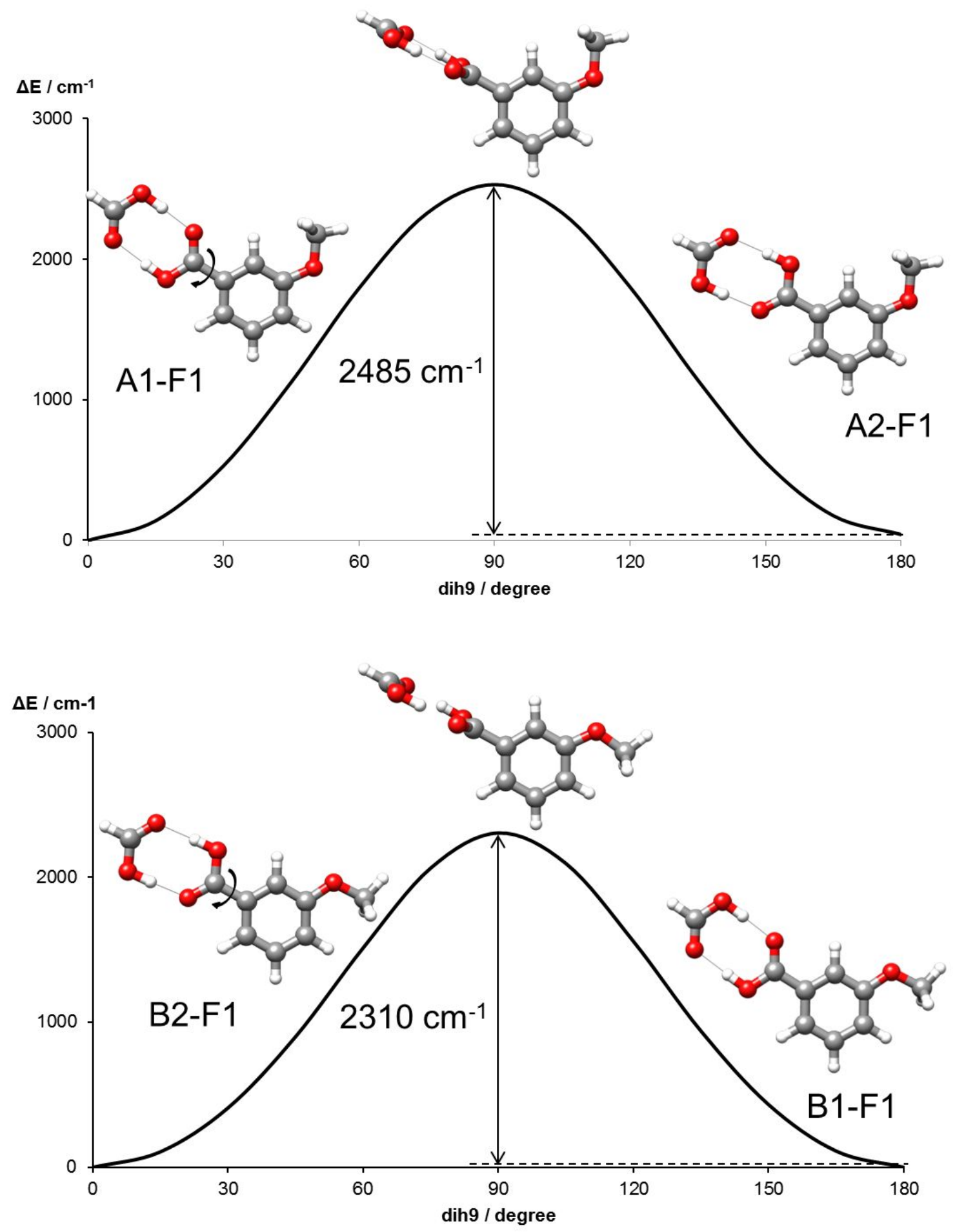
Figure S7. Potential energy functions predicted at MP2/aug-cc-pVDZ level of theory for the interconversion pathway between A1-F1 - B1-F1 and A2-F1 - B2-F1 forms of $m$-anisic acid - formic acid heterodimer through the dihedral angle dih11 $=\mathrm{C}_{2}-\mathrm{C}_{3}-\mathrm{O}_{8}-\mathrm{C}_{11}$ (See Figure $\mathrm{S} 2$ for atom labelling).
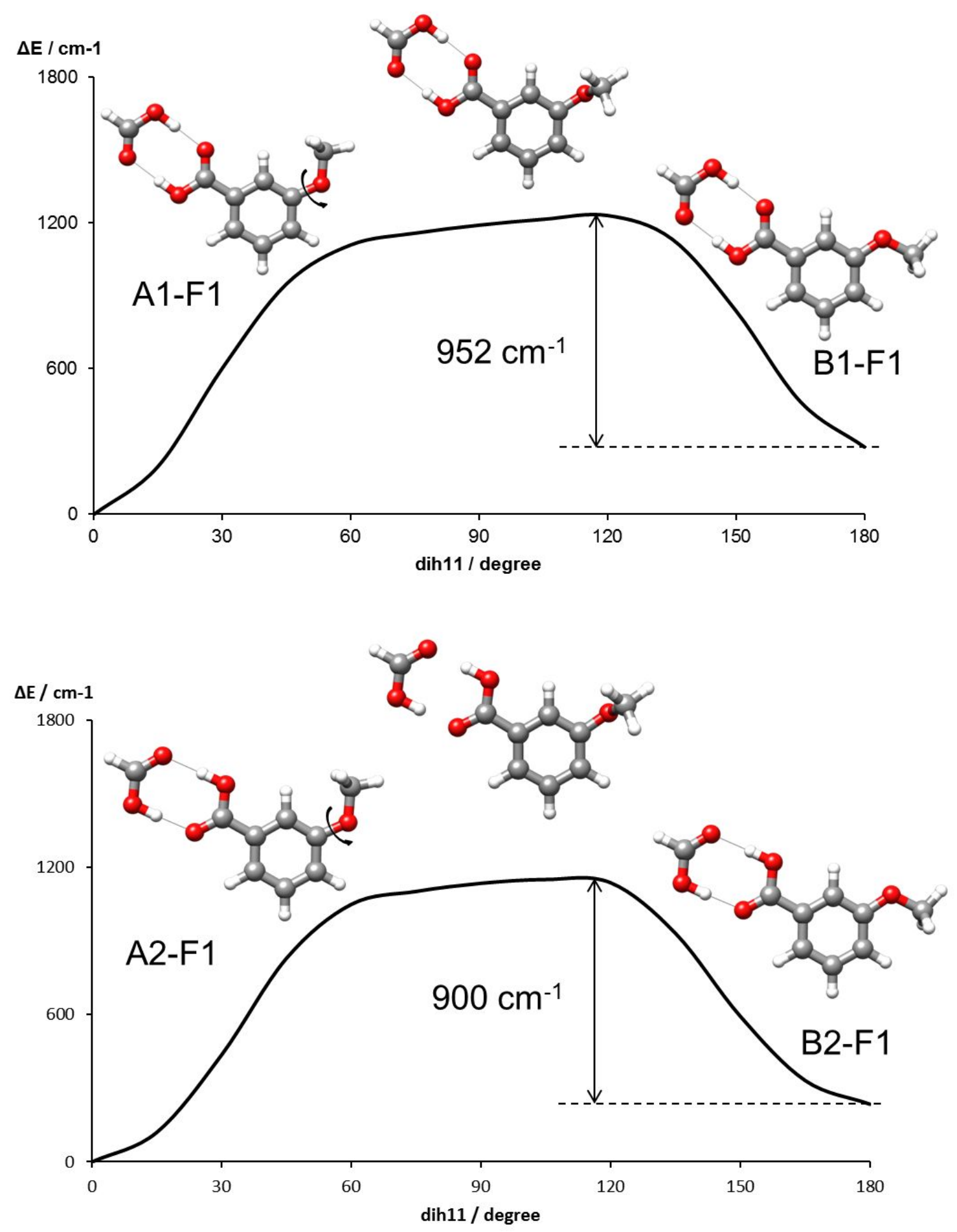
Table S1. Rotational parameters predicted at B3LYP-D3/6-311++G(d,p) level of theory for the stable $m$ anisic acid - formic acid heterodimer conformers (see Figure S2 for notation).

\begin{tabular}{lccccc}
\hline Parameter $^{\mathbf{a}}$ & $\boldsymbol{m}$-ANI A1-F1 & $\boldsymbol{m}$-ANI A2-F1 & $\boldsymbol{m}$-ANI B2-F1 & $\boldsymbol{m}$-ANI B1-F1 & $\boldsymbol{m}$-ANI A2-F2 \\
\hline$A / \mathrm{MHz}$ & 1362.78 & 1451.53 & 1873.73 & 1733.08 & 1245.51 \\
$B / \mathrm{MHz}$ & 300.64 & 293.10 & 262.94 & 268.39 & 324.50 \\
$C / \mathrm{MHz}$ & 246.69 & 244.24 & 230.92 & 232.92 & 257.91 \\
$P_{\mathrm{cc}} / \mathrm{u} \AA^{2}$ & 1.61 & 1.61 & 1.61 & 1.61 & 1.81 \\
$\mu_{\mathrm{a}} / \mathrm{D}$ & 1.3 & 1.3 & 3.2 & -3.3 & -3.0 \\
$\mu_{\mathrm{b}} / \mathrm{D}$ & 1.0 & -0.8 & 0.8 & 0.6 & 2.3 \\
$\mu_{\mathrm{c}} / \mathrm{D}$ & 0.0 & 0.0 & 0.0 & 0.0 & 0.0 \\
$\Delta E_{\mathrm{DFT}} / \mathrm{cm}^{-1}$ & $0.0^{\mathrm{b}}$ & 47.2 & 264.3 & 267.1 & 2802.6 \\
\hline
\end{tabular}

\begin{tabular}{lcccc}
\hline Parameter $^{\mathbf{a}}$ & $\boldsymbol{m}$-ANI B2-F2 & $\boldsymbol{m}$-ANI A1-F2 & $\boldsymbol{m}$-ANI B1-F2 & $\boldsymbol{m}$-ANI B3-F3 \\
\hline$A / \mathrm{MHz}$ & 1231.45 & 1088.99 & 1044.02 & 771.49 \\
$B / \mathrm{MHz}$ & 315.24 & 352.57 & 349.67 & 545.84 \\
$C / \mathrm{MHz}$ & 251.39 & 282.26 & 277.14 & 337.82 \\
$P_{\mathrm{cc}} / \mathrm{u} \AA^{2}$ & 1.62 & 53.50 & 52.92 & 42.47 \\
$\mu_{\mathrm{a}} / \mathrm{D}$ & -3.3 & -1.0 & -1.5 & -7.2 \\
$\mu_{\mathrm{b}} / \mathrm{D}$ & 0.3 & 0.4 & -2.0 & -3.5 \\
$\mu_{\mathrm{c}} / \mathrm{D}$ & 0.0 & 0.9 & 0.6 & 1.1 \\
$\Delta E_{\mathrm{DFT}} / \mathrm{cm}^{-1}$ & 2932.6 & 3775.6 & 4054.6 & 4383.4 \\
\hline${ }^{\mathrm{a}} A, B$ and $C$ are the rotational constants. $\mu_{\mathrm{a},} \mu_{\mathrm{b}}$ and $\mu_{\mathrm{c}}$ are the components of the electric dipole moment. \\
$P_{\mathrm{cc}}$ is the planar moment of inertia in the $a b$ plane, derived from $P_{\mathrm{cc}}=\left(I_{\mathrm{a}}+I_{\mathrm{b}}-I_{\mathrm{c}}\right) / 2 . \Delta E$ is the energy relative \\
to conformer $m$-ANI A1-F1. ${ }^{\mathrm{b}}$ Absolute energy is $-725.3771589 \mathrm{E}_{\mathrm{h}}$.
\end{tabular}


Table S2. Predicted $r_{\mathrm{e}}$ geometry (lengths in $\AA$ and angles in degree) calculated at B3LYP-D3/ $6-311++\mathrm{G}(\mathrm{d}, \mathrm{p})$ level of theory for the conformers of $m$-anisic acid molecule. See Figure $\mathrm{S} 1$ for atom labelling.

\begin{tabular}{|c|c|c|c|c|}
\hline Parameter & $m$-ANI A1 $r_{\mathrm{e}}$ & $m$-ANI A2 $\mathrm{r}_{\mathrm{e}}$ & $m$-ANI B2 $r_{\mathrm{e}}$ & $m$-ANI B1 $r_{\mathrm{e}}$ \\
\hline $\mathrm{r}\left(\mathrm{C}_{1}-\mathrm{C}_{2}\right)$ & 1.402 & 1.403 & 1.393 & 1.392 \\
\hline $\mathrm{r}\left(\mathrm{C}_{2}-\mathrm{C}_{3}\right)$ & 1.393 & 1.394 & 1.399 & 1.397 \\
\hline $\mathrm{r}\left(\mathrm{C}_{3}-\mathrm{C}_{4}\right)$ & 1.402 & 1.401 & 1.398 & 1.399 \\
\hline $\mathrm{r}\left(\mathrm{C}_{4}-\mathrm{C}_{5}\right)$ & 1.387 & 1.388 & 1.398 & 1.397 \\
\hline $\mathrm{r}\left(\mathrm{C}_{5}-\mathrm{C}_{6}\right)$ & 1.395 & 1.394 & 1.386 & 1.388 \\
\hline $\mathrm{r}\left(\mathrm{C}_{1}-\mathrm{C}_{6}\right)$ & 1.395 & 1.394 & 1.402 & 1.403 \\
\hline $\mathrm{r}\left(\mathrm{C}_{1}-\mathrm{C}_{7}\right)$ & 1.487 & 1.487 & 1.488 & 1.488 \\
\hline $\mathrm{r}\left(\mathrm{C}_{3}-\mathrm{O}_{8}\right)$ & 1.362 & 1.363 & 1.363 & 1.362 \\
\hline $\mathrm{r}\left(\mathrm{C}_{7}-\mathrm{O}_{9}\right)^{\mathrm{a}}$ & 1.358 & 1.361 & 1.358 & 1.359 \\
\hline $\mathrm{r}\left(\mathrm{C}_{7}-\mathrm{O}_{10}\right)^{\mathrm{a}}$ & 1.210 & 1.209 & 1.209 & 1.209 \\
\hline $\mathrm{r}\left(\mathrm{O}_{8}-\mathrm{C}_{11}\right)$ & 1.424 & 1.423 & 1.422 & 1.422 \\
\hline $\mathrm{r}\left(\mathrm{O}_{9}-\mathrm{H}_{16}\right)$ & 0.968 & 0.968 & 0.968 & 0.968 \\
\hline$\angle\left(\mathrm{C}_{1}-\mathrm{C}_{2}-\mathrm{C}_{3}\right)$ & 119.5 & 119.4 & 119.9 & 120.1 \\
\hline$\angle\left(\mathrm{C}_{2}-\mathrm{C}_{3}-\mathrm{C}_{4}\right)$ & 119.8 & 119.9 & 119.8 & 119.7 \\
\hline$\angle\left(\mathrm{C}_{3}-\mathrm{C}_{4}-\mathrm{C}_{5}\right)$ & 120.2 & 120.2 & 119.6 & 119.6 \\
\hline$\angle\left(\mathrm{C}_{4}-\mathrm{C}_{5}-\mathrm{C}_{6}\right)$ & 120.7 & 120.5 & 121.0 & 120.5 \\
\hline$\angle\left(\mathrm{C}_{1}-\mathrm{C}_{6}-\mathrm{C}_{5}\right)$ & 119.0 & 119.2 & 119.2 & 119.0 \\
\hline$\angle\left(\mathrm{C}_{2}-\mathrm{C}_{1}-\mathrm{C}_{6}\right)$ & 120.8 & 120.8 & 120.5 & 118.8 \\
\hline$\angle\left(\mathrm{C}_{2}-\mathrm{C}_{1}-\mathrm{C}_{7}\right)$ & 117.1 & 121.3 & 121.6 & 117.4 \\
\hline$\angle\left(\mathrm{C}_{6}-\mathrm{C}_{1}-\mathrm{C}_{7}\right)$ & 122.1 & 117.9 & 117.9 & 122.1 \\
\hline$\angle\left(\mathrm{C}_{2}-\mathrm{C}_{3}-\mathrm{O}_{8}\right)$ & 124.5 & 124.5 & 115.5 & 115.6 \\
\hline$\angle\left(\mathrm{C}_{4}-\mathrm{C}_{3}-\mathrm{O}_{8}\right)$ & 115.7 & 115.6 & 124.7 & 124.6 \\
\hline$\angle\left(\mathrm{C}_{1}-\mathrm{C}_{7}-\mathrm{O}_{9}\right)$ & 113.1 & 113.1 & 113.1 & 112.9 \\
\hline$\angle\left(\mathrm{C}_{1}-\mathrm{C}_{7}-\mathrm{O}_{10}\right)$ & 125.0 & 125.1 & 124.8 & 125.1 \\
\hline$\angle\left(\mathrm{C}_{3}-\mathrm{O}_{8}-\mathrm{C}_{11}\right)$ & 120.2 & 118.6 & 118.7 & 118.7 \\
\hline$\angle\left(\mathrm{C}_{7}-\mathrm{O}_{9}-\mathrm{H}_{16}\right)$ & 106.8 & 106.7 & 106.9 & 106.8 \\
\hline$\angle\left(\mathrm{C}_{2}-\mathrm{C}_{1}-\mathrm{C}_{7}-\mathrm{O}_{9}\right)$ & 180.0 & 0.0 & 0.0 & 180.0 \\
\hline$\angle\left(\mathrm{C}_{2}-\mathrm{C}_{3}-\mathrm{O}_{8}-\mathrm{C}_{11}\right)$ & 0.0 & 0.0 & 180.0 & 180.0 \\
\hline
\end{tabular}

a The predicted values of these bond lengths at the same level of theory for the formic acid molecule were $1.346 \AA$ and $1.199 \AA$ respectively. 
Table S3. Predicted $r_{\mathrm{e}}$ geometry (lengths in $\AA$ and angles in degree) calculated at B3LYP-D3/ $6-311++\mathrm{G}(\mathrm{d}, \mathrm{p})$ level of theory for the conformers of $m$-anisic acid - formic acid cluster. See Figure S2 for atom labelling.

\begin{tabular}{|c|c|c|c|c|}
\hline Parameter & $m$-ANI A1-F1 $r_{\mathrm{e}}$ & $m$-ANI A2-F1 $\mathrm{r}_{\mathrm{e}}$ & $m$-ANI B2-F1 $\mathbf{r}_{\mathrm{e}}$ & $m$-ANI B1-F1 $r_{\mathrm{e}}$ \\
\hline $\mathrm{r}\left(\mathrm{C}_{1}-\mathrm{C}_{2}\right)$ & 1.402 & 1.403 & 1.393 & 1.393 \\
\hline $\mathrm{r}\left(\mathrm{C}_{2}-\mathrm{C}_{3}\right)$ & 1.393 & 1.394 & 1.398 & 1.397 \\
\hline $\mathrm{r}\left(\mathrm{C}_{3}-\mathrm{C}_{4}\right)$ & 1.402 & 1.402 & 1.398 & 1.399 \\
\hline $\mathrm{r}\left(\mathrm{C}_{4}-\mathrm{C}_{5}\right)$ & 1.388 & 1.388 & 1.398 & 1.397 \\
\hline $\mathrm{r}\left(\mathrm{C}_{5}-\mathrm{C}_{6}\right)$ & 1.395 & 1.394 & 1.386 & 1.387 \\
\hline $\mathrm{r}\left(\mathrm{C}_{1}-\mathrm{C}_{6}\right)$ & 1.395 & 1.395 & 1.402 & 1.403 \\
\hline $\mathrm{r}\left(\mathrm{C}_{1}-\mathrm{C}_{7}\right)$ & 1.485 & 1.485 & 1.486 & 1.486 \\
\hline $\mathrm{r}\left(\mathrm{C}_{3}-\mathrm{O}_{8}\right)$ & 1.362 & 1.362 & 1.362 & 1.362 \\
\hline $\mathrm{r}\left(\mathrm{C}_{7}-\mathrm{O}_{9}\right)$ & 1.323 & 1.325 & 1.323 & 1.324 \\
\hline $\mathrm{r}\left(\mathrm{C}_{7}-\mathrm{O}_{10}\right)$ & 1.231 & 1.230 & 1.230 & 1.230 \\
\hline $\mathrm{r}\left(\mathrm{O}_{8}-\mathrm{C}_{11}\right)$ & 1.424 & 1.424 & 1.423 & 1.423 \\
\hline $\mathrm{r}\left(\mathrm{O}_{9}-\mathrm{O}_{12}\right)$ & 2.694 & 2.691 & 2.695 & 2.693 \\
\hline $\mathrm{r}\left(\mathrm{O}_{10}-\mathrm{O}_{14}\right)$ & 2.647 & 2.647 & 2.647 & 2.647 \\
\hline $\mathrm{r}\left(\mathrm{O}_{9}-\mathrm{H}_{19}\right)$ & 0.995 & 0.995 & 0.995 & 0.995 \\
\hline $\mathrm{r}\left(\mathrm{O}_{12}-\mathrm{H}_{19}\right)$ & 1.670 & 1.696 & 1.700 & 1.699 \\
\hline $\mathrm{r}\left(\mathrm{O}_{14}-\mathrm{H}_{23}\right)$ & 1.004 & 1.004 & 1.004 & 1.004 \\
\hline $\mathrm{r}\left(\mathrm{O}_{10}-\mathrm{H}_{23}\right)$ & 1.643 & 1.643 & 1.643 & 1.643 \\
\hline$\angle\left(\mathrm{C}_{1}-\mathrm{C}_{2}-\mathrm{C}_{3}\right)$ & 119.5 & 119.4 & 119.9 & 120.1 \\
\hline$\angle\left(\mathrm{C}_{2}-\mathrm{C}_{3}-\mathrm{C}_{4}\right)$ & 119.7 & 119.8 & 119.8 & 119.7 \\
\hline$\angle\left(\mathrm{C}_{3}-\mathrm{C}_{4}-\mathrm{C}_{5}\right)$ & 120.2 & 120.2 & 119.7 & 119.7 \\
\hline$\angle\left(\mathrm{C}_{4}-\mathrm{C}_{5}-\mathrm{C}_{6}\right)$ & 120.6 & 120.6 & 121.0 & 121.0 \\
\hline$\angle\left(\mathrm{C}_{1}-\mathrm{C}_{6}-\mathrm{C}_{5}\right)$ & 119.0 & 119.2 & 119.1 & 119.0 \\
\hline$\angle\left(\mathrm{C}_{2}-\mathrm{C}_{1}-\mathrm{C}_{6}\right)$ & 120.9 & 120.8 & 120.5 & 120.5 \\
\hline$\angle\left(\mathrm{C}_{2}-\mathrm{C}_{1}-\mathrm{C}_{7}\right)$ & 117.8 & 120.5 & 120.9 & 118.1 \\
\hline$\angle\left(\mathrm{C}_{6}-\mathrm{C}_{1}-\mathrm{C}_{7}\right)$ & 121.3 & 118.7 & 118.6 & 121.3 \\
\hline$\angle\left(\mathrm{C}_{2}-\mathrm{C}_{3}-\mathrm{O}_{8}\right)$ & 124.5 & 124.5 & 115.5 & 115.6 \\
\hline$\angle\left(\mathrm{C}_{4}-\mathrm{C}_{3}-\mathrm{O}_{8}\right)$ & 115.7 & 115.7 & 124.7 & 124.7 \\
\hline$\angle\left(\mathrm{C}_{1}-\mathrm{C}_{7}-\mathrm{O}_{9}\right)$ & 114.5 & 114.5 & 114.6 & 114.3 \\
\hline$\angle\left(\mathrm{C}_{1}-\mathrm{C}_{7}-\mathrm{O}_{10}\right)$ & 122.2 & 122.3 & 122.1 & 122.3 \\
\hline$\angle\left(\mathrm{C}_{3}-\mathrm{O}_{8}-\mathrm{C}_{11}\right)$ & 118.5 & 118.5 & 118.7 & 118.7 \\
\hline$\angle\left(\mathrm{C}_{7}-\mathrm{O}_{9}-\mathrm{H}_{19}\right)$ & 110.5 & 110.5 & 110.5 & 110.5 \\
\hline$\angle\left(\mathrm{C}_{7}-\mathrm{O}_{10}-\mathrm{H}_{23}\right)$ & 128.3 & 128.3 & 128.3 & 128.3 \\
\hline$\angle\left(\mathrm{O}_{9}-\mathrm{H}_{19}-\mathrm{O}_{12}\right)$ & 178.5 & 178.6 & 178.3 & 178.5 \\
\hline$\angle\left(\mathrm{O}_{10}-\mathrm{H}_{23}-\mathrm{O}_{14}\right)$ & 178.0 & 177.8 & 177.9 & 177.8 \\
\hline$\angle\left(\mathrm{C}_{2}-\mathrm{C}_{1}-\mathrm{C}_{7}-\mathrm{O}_{9}\right)$ & 180.0 & 0.0 & 0.0 & 180.0 \\
\hline$\angle\left(\mathrm{C}_{2}-\mathrm{C}_{3}-\mathrm{O}_{8}-\mathrm{C}_{11}\right)$ & 0.0 & 0.0 & 180.0 & 180.0 \\
\hline
\end{tabular}


Table S4. Observed rotational transitions and residuals (all the values in $\mathrm{MHz}$ ) for the A1 conformation of the $m$-anisic acid in the ground vibrational state.

\begin{tabular}{|c|c|c|c|c|c|c|c|}
\hline $\mathbf{J}^{\prime}$ & $\mathbf{K}_{-1}^{\prime}$ & $\mathbf{K}_{+1}^{\prime}$ & $\mathrm{J}^{\prime \prime}$ & $\mathbf{K}_{-1}{ }^{\prime \prime}$ & $\mathbf{K}_{+1}{ }^{\prime \prime}$ & Obs. & Obs.-Cal. \\
\hline 1 & 1 & 1 & 0 & 0 & 0 & 2458.4745 & 0.0016 \\
\hline 2 & 0 & 2 & 1 & 0 & 1 & 2640.1710 & -0.0122 \\
\hline 2 & 1 & 2 & 1 & 0 & 1 & 3569.3708 & 0.0000 \\
\hline 2 & 2 & 0 & 1 & 1 & 1 & 6519.2880 & 0.0046 \\
\hline 2 & 2 & 1 & 1 & 1 & 0 & 6264.5195 & -0.0011 \\
\hline 3 & 1 & 3 & 2 & 1 & 2 & 3651.4124 & -0.0005 \\
\hline 3 & 0 & 3 & 2 & 0 & 2 & 3887.8506 & 0.0036 \\
\hline 3 & 1 & 2 & 2 & 1 & 1 & 4321.9594 & -0.0036 \\
\hline 3 & 2 & 1 & 2 & 2 & 0 & 4123.8506 & 0.0111 \\
\hline 3 & 2 & 2 & 2 & 2 & 1 & 4005.8467 & 0.0031 \\
\hline 3 & 0 & 3 & 2 & 1 & 2 & 2958.6565 & -0.0028 \\
\hline 3 & 1 & 3 & 2 & 0 & 2 & 4580.6015 & 0.0009 \\
\hline 3 & 2 & 2 & 2 & 1 & 1 & 7375.4193 & 0.0011 \\
\hline 3 & 1 & 2 & 3 & 0 & 3 & 2036.4393 & -0.0144 \\
\hline 3 & 2 & 1 & 3 & 1 & 2 & 3201.8218 & -0.0085 \\
\hline 3 & 2 & 2 & 3 & 1 & 3 & 4397.1484 & -0.0067 \\
\hline 3 & 3 & 0 & 3 & 2 & 1 & 6048.9988 & 0.0026 \\
\hline 3 & 3 & 1 & 3 & 2 & 2 & 6194.7724 & 0.0002 \\
\hline 4 & 1 & 4 & 3 & 1 & 3 & 4838.6183 & -0.0031 \\
\hline 4 & 0 & 4 & 3 & 0 & 3 & 5065.8305 & -0.0024 \\
\hline 4 & 1 & 3 & 3 & 1 & 2 & 5720.7476 & -0.0005 \\
\hline 4 & 2 & 2 & 3 & 2 & 1 & 5592.4866 & -0.0054 \\
\hline 4 & 2 & 3 & 3 & 2 & 2 & 5317.3914 & 0.0040 \\
\hline 4 & 3 & 1 & 3 & 3 & 0 & 5410.2742 & 0.0109 \\
\hline 4 & 3 & 2 & 3 & 3 & 1 & 5394.8686 & 0.0099 \\
\hline 4 & 0 & 4 & 3 & 1 & 3 & 4373.0761 & -0.0031 \\
\hline 4 & 1 & 4 & 3 & 0 & 3 & 5531.3771 & 0.0019 \\
\hline 4 & 1 & 3 & 4 & 0 & 4 & 2691.3634 & -0.0057 \\
\hline 4 & 2 & 2 & 4 & 1 & 3 & 3073.5750 & 0.0009 \\
\hline 4 & 2 & 3 & 4 & 1 & 4 & 4875.9324 & 0.0114 \\
\hline 4 & 3 & 1 & 4 & 2 & 2 & 5866.7655 & -0.0019 \\
\hline 4 & 3 & 2 & 4 & 2 & 3 & 6272.2430 & -0.0005 \\
\hline 5 & 1 & 5 & 4 & 1 & 4 & 6006.7339 & -0.0004 \\
\hline 5 & 0 & 5 & 4 & 0 & 4 & 6186.8635 & -0.0017 \\
\hline 5 & 1 & 4 & 4 & 1 & 3 & 7076.1338 & -0.0020 \\
\hline 5 & 2 & 3 & 4 & 2 & 2 & 7097.0751 & 0.0044 \\
\hline 5 & 2 & 4 & 4 & 2 & 3 & 6608.9975 & -0.0010 \\
\hline
\end{tabular}


Table S4 (Continued).

\begin{tabular}{|c|c|c|c|c|c|c|c|}
\hline $\mathbf{J}^{\prime}$ & $\mathrm{K}_{-1}^{\prime}$ & $\mathbf{K}_{+1}^{\prime}$ & $\mathbf{J}^{\prime \prime}$ & $\mathbf{K}_{-1}{ }^{\prime \prime}$ & $\mathbf{K}_{+1}^{\prime \prime}$ & Obs. & Obs.-Cal. \\
\hline 5 & 3 & 2 & 4 & 3 & 1 & 6808.8901 & -0.0076 \\
\hline 5 & 3 & 3 & 4 & 3 & 2 & 6756.3943 & -0.0045 \\
\hline 5 & 4 & 1 & 4 & 4 & 0 & 6745.2717 & 0.0051 \\
\hline 5 & 4 & 2 & 4 & 4 & 1 & 6743.8023 & -0.0027 \\
\hline 5 & 0 & 5 & 4 & 1 & 4 & 5721.3278 & 0.0048 \\
\hline 5 & 1 & 5 & 4 & 0 & 4 & 6472.2771 & 0.0005 \\
\hline 5 & 1 & 4 & 4 & 2 & 3 & 4426.0425 & 0.0008 \\
\hline 5 & 1 & 4 & 5 & 0 & 5 & 3580.6480 & 0.0083 \\
\hline 5 & 2 & 3 & 5 & 1 & 4 & 3094.5161 & 0.0072 \\
\hline 5 & 2 & 4 & 5 & 1 & 5 & 5478.1818 & -0.0034 \\
\hline 5 & 3 & 2 & 5 & 2 & 3 & 5578.6047 & 0.0100 \\
\hline 5 & 3 & 3 & 5 & 2 & 4 & 6419.6435 & -0.0002 \\
\hline 6 & 1 & 6 & 5 & 1 & 5 & 7157.3757 & -0.0022 \\
\hline 6 & 0 & 6 & 5 & 0 & 5 & 7279.9403 & -0.0003 \\
\hline 6 & 1 & 5 & 5 & 1 & 4 & 8370.4809 & 0.0067 \\
\hline 6 & 2 & 4 & 5 & 2 & 3 & 8601.9384 & -0.0105 \\
\hline 6 & 2 & 5 & 5 & 2 & 4 & 7876.6856 & -0.0143 \\
\hline 6 & 3 & 4 & 5 & 3 & 3 & 8116.2775 & -0.0015 \\
\hline 6 & 0 & 6 & 5 & 1 & 5 & 6994.5282 & -0.0011 \\
\hline 6 & 1 & 6 & 5 & 0 & 5 & 7442.7904 & 0.0011 \\
\hline 6 & 2 & 4 & 5 & 3 & 3 & 3093.8648 & 0.0076 \\
\hline 6 & 1 & 5 & 6 & 0 & 6 & 4671.1787 & 0.0055 \\
\hline 6 & 2 & 4 & 6 & 1 & 5 & 3325.9919 & 0.0082 \\
\hline 6 & 2 & 5 & 6 & 1 & 6 & 6197.4990 & -0.0083 \\
\hline 6 & 3 & 3 & 6 & 2 & 4 & 5226.0056 & 0.0023 \\
\hline 6 & 3 & 4 & 6 & 2 & 5 & 6659.2155 & -0.0073 \\
\hline 7 & 1 & 7 & 6 & 1 & 6 & 8293.9275 & 0.0049 \\
\hline 7 & 1 & 6 & 6 & 1 & 5 & 9589.5818 & -0.0048 \\
\hline 7 & 2 & 6 & 6 & 2 & 5 & 9117.8016 & -0.0035 \\
\hline 7 & 4 & 3 & 6 & 4 & 2 & 9511.4146 & 0.0014 \\
\hline 7 & 0 & 7 & 6 & 1 & 6 & 8205.7416 & -0.0017 \\
\hline 7 & 1 & 6 & 6 & 2 & 5 & 7900.4093 & 0.0053 \\
\hline 7 & 2 & 5 & 7 & 1 & 6 & 3811.4576 & 0.0051 \\
\hline 7 & 2 & 6 & 7 & 1 & 7 & 7021.3964 & 0.0065 \\
\hline 7 & 3 & 4 & 7 & 2 & 5 & 4892.6872 & -0.0045 \\
\hline 7 & 3 & 5 & 7 & 2 & 6 & 7008.7981 & 0.0045 \\
\hline 8 & 1 & 8 & 7 & 1 & 7 & 9420.2732 & 0.0037 \\
\hline 8 & 0 & 8 & 7 & 0 & 7 & 9462.4692 & 0.0008 \\
\hline
\end{tabular}


Table S4 (Continued).

\begin{tabular}{rrrrrrrr}
\hline $\mathbf{J}^{\prime}$ & $\mathbf{K}_{\mathbf{- 1}}{ }^{\prime}$ & $\mathbf{K}_{+\mathbf{1}}{ }^{\prime}$ & $\mathbf{J}^{\prime \prime}$ & $\mathbf{K}_{-\mathbf{1}}{ }^{\prime \prime}$ & $\mathbf{K}_{+\mathbf{1}}{ }^{\prime \prime}$ & \multicolumn{1}{l}{ Obs. } & \multicolumn{1}{c}{ Obs.-Cal. } \\
\hline 8 & 1 & 8 & 7 & 0 & 7 & 9508.4470 & -0.0015 \\
8 & 2 & 6 & 8 & 1 & 7 & 4572.9639 & -0.0052 \\
8 & 3 & 5 & 8 & 2 & 6 & 4678.5431 & 0.0059 \\
8 & 4 & 4 & 8 & 3 & 5 & 7806.3715 & -0.0010 \\
9 & 3 & 6 & 9 & 2 & 7 & 4672.3486 & 0.0013 \\
9 & 4 & 5 & 9 & 3 & 6 & 7349.7342 & -0.0095 \\
10 & 3 & 7 & 10 & 3 & 8 & 2669.3858 & -0.0044 \\
10 & 2 & 8 & 10 & 1 & 9 & 6822.3460 & -0.0016 \\
10 & 3 & 7 & 10 & 2 & 8 & 4937.9141 & -0.0061 \\
10 & 4 & 6 & 10 & 3 & 7 & 6854.1958 & 0.0020 \\
11 & 3 & 8 & 11 & 2 & 9 & 5512.3048 & -0.0085 \\
11 & 4 & 7 & 11 & 3 & 8 & 6422.1877 & 0.0066 \\
12 & 4 & 8 & 12 & 3 & 9 & 6164.0043 & -0.0040 \\
12 & 5 & 7 & 12 & 4 & 8 & 9559.7389 & 0.0066 \\
14 & 4 & 10 & 14 & 4 & 11 & 3785.4080 & 0.0069 \\
14 & 6 & 8 & 13 & 7 & 7 & 3548.7777 & 0.0002 \\
18 & 5 & 13 & 18 & 4 & 14 & 8056.4949 & -0.0009 \\
\hline
\end{tabular}


Table S5. Observed rotational transitions and residuals (all the values in $\mathrm{MHz}$ ) for the A2 conformation of the $m$-anisic acid in the ground vibrational state.

\begin{tabular}{|c|c|c|c|c|c|c|c|}
\hline $\mathbf{J}^{\prime}$ & $\mathrm{K}_{-1}{ }^{\prime}$ & $\mathbf{K}_{+1}^{\prime}$ & $\mathbf{J}^{\prime \prime}$ & $\mathbf{K}_{-1}{ }^{\prime \prime}$ & $\mathbf{K}_{+1}{ }^{\prime \prime}$ & Obs. & Obs.-Cal. \\
\hline 1 & 0 & 1 & 0 & 0 & 0 & 1350.6887 & -0.0095 \\
\hline 1 & 1 & 1 & 0 & 0 & 0 & 2419.0247 & -0.0077 \\
\hline 2 & 1 & 2 & 1 & 1 & 1 & 2467.0035 & -0.0012 \\
\hline 2 & 0 & 2 & 1 & 0 & 1 & 2666.8878 & -0.0030 \\
\hline 2 & 1 & 2 & 1 & 0 & 1 & 3535.3345 & -0.0044 \\
\hline 2 & 2 & 0 & 1 & 1 & 1 & 6409.6859 & 0.0003 \\
\hline 2 & 2 & 1 & 1 & 1 & 0 & 6140.7889 & 0.0000 \\
\hline 2 & 2 & 0 & 2 & 1 & 1 & 3239.5082 & 0.0014 \\
\hline 2 & 2 & 1 & 2 & 1 & 2 & 3908.1734 & -0.0023 \\
\hline 3 & 1 & 3 & 2 & 1 & 2 & 3680.3230 & -0.0001 \\
\hline 3 & 0 & 3 & 2 & 0 & 2 & 3918.7567 & -0.0008 \\
\hline 3 & 1 & 2 & 2 & 1 & 1 & 4380.2782 & -0.0015 \\
\hline 3 & 2 & 1 & 2 & 2 & 0 & 4185.4285 & -0.0001 \\
\hline 3 & 2 & 2 & 2 & 2 & 1 & 4052.0918 & -0.0019 \\
\hline 3 & 0 & 3 & 2 & 1 & 2 & 3050.3043 & -0.0050 \\
\hline 3 & 1 & 3 & 2 & 0 & 2 & 4548.7703 & -0.0010 \\
\hline 3 & 2 & 1 & 2 & 1 & 2 & 8128.1079 & -0.0015 \\
\hline 3 & 2 & 2 & 2 & 1 & 1 & 7257.0960 & 0.0007 \\
\hline 3 & 3 & 0 & 2 & 2 & 1 & 10003.1358 & -0.0001 \\
\hline 3 & 3 & 1 & 2 & 2 & 0 & 9965.4123 & -0.0021 \\
\hline 3 & 1 & 2 & 3 & 0 & 3 & 2033.1389 & -0.0056 \\
\hline 3 & 2 & 1 & 3 & 1 & 2 & 3044.6556 & 0.0000 \\
\hline 3 & 2 & 2 & 3 & 1 & 3 & 4279.9486 & 0.0022 \\
\hline 3 & 3 & 0 & 3 & 2 & 1 & 5783.2015 & -0.0007 \\
\hline 3 & 3 & 1 & 3 & 2 & 2 & 5947.8268 & 0.0010 \\
\hline 4 & 1 & 4 & 3 & 1 & 3 & 4873.5989 & 0.0000 \\
\hline 4 & 0 & 4 & 3 & 0 & 3 & 5095.0053 & -0.0018 \\
\hline 4 & 1 & 3 & 3 & 1 & 2 & 5792.1416 & -0.0018 \\
\hline 4 & 2 & 2 & 3 & 2 & 1 & 5683.3406 & 0.0001 \\
\hline 4 & 2 & 3 & 3 & 2 & 2 & 5375.8062 & -0.0019 \\
\hline 4 & 3 & 1 & 3 & 3 & 0 & 5482.2143 & -0.0026 \\
\hline 4 & 3 & 2 & 3 & 3 & 1 & 5463.1975 & -0.0013 \\
\hline 4 & 1 & 3 & 4 & 1 & 4 & 2321.6691 & -0.0061 \\
\hline 4 & 2 & 3 & 4 & 0 & 4 & 5190.7408 & -0.0202 \\
\hline 4 & 0 & 4 & 3 & 1 & 3 & 4464.9917 & -0.0016 \\
\hline 4 & 1 & 4 & 3 & 0 & 3 & 5503.6126 & -0.0001 \\
\hline 4 & 1 & 3 & 3 & 2 & 2 & 2915.3142 & -0.0136 \\
\hline
\end{tabular}


Table S5 (Continued).

\begin{tabular}{|c|c|c|c|c|c|c|c|}
\hline $\mathbf{J}^{\prime}$ & $\mathrm{K}_{-1}^{\prime}$ & $\mathbf{K}_{+1}^{\prime}$ & $\mathbf{J}^{\prime \prime}$ & $\mathbf{K}_{-1}{ }^{\prime \prime}$ & $\mathbf{K}_{+1}^{\prime \prime}$ & Obs. & Obs.-Cal. \\
\hline 4 & 2 & 2 & 3 & 1 & 3 & 10131.1266 & -0.0001 \\
\hline 4 & 2 & 3 & 3 & 1 & 2 & 8252.6219 & -0.0017 \\
\hline 4 & 3 & 1 & 3 & 2 & 2 & 11433.2598 & 0.0005 \\
\hline 4 & 3 & 2 & 3 & 2 & 1 & 11243.1851 & 0.0003 \\
\hline 4 & 1 & 3 & 4 & 0 & 4 & 2730.2793 & -0.0015 \\
\hline 4 & 2 & 3 & 4 & 1 & 4 & 4782.1567 & 0.0012 \\
\hline 4 & 3 & 1 & 4 & 2 & 2 & 5582.0800 & 0.0011 \\
\hline 4 & 3 & 2 & 4 & 2 & 3 & 6035.2182 & 0.0015 \\
\hline 4 & 4 & 1 & 4 & 3 & 2 & 8245.0824 & 0.0037 \\
\hline 5 & 1 & 5 & 4 & 1 & 4 & 6046.1125 & -0.0007 \\
\hline 5 & 0 & 5 & 4 & 0 & 4 & 6214.0333 & -0.0008 \\
\hline 5 & 1 & 4 & 4 & 1 & 3 & 7153.5693 & 0.0002 \\
\hline 5 & 2 & 3 & 4 & 2 & 2 & 7214.4290 & -0.0004 \\
\hline 5 & 2 & 4 & 4 & 2 & 3 & 6676.9491 & -0.0007 \\
\hline 5 & 3 & 2 & 4 & 3 & 1 & 6906.6950 & -0.0017 \\
\hline 5 & 3 & 3 & 4 & 3 & 2 & 6842.2194 & 0.0000 \\
\hline 5 & 4 & 1 & 4 & 4 & 0 & 6831.9855 & -0.0024 \\
\hline 5 & 4 & 2 & 4 & 4 & 1 & 6830.0207 & -0.0004 \\
\hline 5 & 1 & 4 & 5 & 1 & 5 & 3429.1276 & -0.0034 \\
\hline 5 & 2 & 4 & 5 & 0 & 5 & 5653.6661 & -0.0107 \\
\hline 5 & 0 & 5 & 4 & 1 & 4 & 5805.4293 & 0.0006 \\
\hline 5 & 1 & 5 & 4 & 0 & 4 & 6454.7182 & -0.0005 \\
\hline 5 & 1 & 4 & 4 & 2 & 3 & 4693.0874 & -0.0013 \\
\hline 5 & 2 & 4 & 4 & 1 & 3 & 9137.4295 & -0.0006 \\
\hline 5 & 3 & 2 & 4 & 2 & 3 & 12964.1444 & -0.0034 \\
\hline 5 & 3 & 3 & 4 & 2 & 2 & 12402.0545 & -0.0092 \\
\hline 5 & 1 & 4 & 5 & 0 & 5 & 3669.8144 & -0.0012 \\
\hline 5 & 2 & 3 & 5 & 1 & 4 & 2996.7166 & 0.0035 \\
\hline 5 & 2 & 4 & 5 & 1 & 5 & 5412.9935 & 0.0012 \\
\hline 5 & 3 & 2 & 5 & 2 & 3 & 5274.3463 & 0.0001 \\
\hline 5 & 3 & 3 & 5 & 2 & 4 & 6200.4849 & -0.0011 \\
\hline 5 & 4 & 1 & 5 & 3 & 2 & 8148.3778 & -0.0057 \\
\hline 5 & 4 & 2 & 5 & 3 & 3 & 8232.8752 & -0.0051 \\
\hline 6 & 1 & 6 & 5 & 1 & 5 & 7200.2026 & -0.0011 \\
\hline 6 & 0 & 6 & 5 & 0 & 5 & 7309.0996 & 0.0004 \\
\hline 6 & 1 & 5 & 5 & 1 & 4 & 8444.5704 & -0.0001 \\
\hline 6 & 2 & 4 & 5 & 2 & 3 & 8737.5867 & 0.0003 \\
\hline 6 & 2 & 5 & 5 & 2 & 4 & 7951.2180 & 0.0015 \\
\hline
\end{tabular}


Table S5 (Continued).

\begin{tabular}{|c|c|c|c|c|c|c|c|}
\hline $\mathbf{J}^{\prime}$ & $\mathrm{K}_{-1}^{\prime}$ & $\mathbf{K}_{+1}^{\prime}$ & $\mathbf{J}^{\prime \prime}$ & $\mathbf{K}_{-1}{ }^{\prime \prime}$ & $\mathbf{K}_{+1}{ }^{\prime \prime}$ & Obs. & Obs.-Cal. \\
\hline 6 & 3 & 3 & 5 & 3 & 2 & 8379.7115 & 0.0004 \\
\hline 6 & 3 & 4 & 5 & 3 & 3 & 8217.9118 & -0.0011 \\
\hline 6 & 4 & 3 & 5 & 3 & 3 & 8218.8107 & -0.0002 \\
\hline 6 & 4 & 2 & 5 & 4 & 2 & 8227.5090 & -0.0030 \\
\hline 6 & 1 & 5 & 5 & 4 & 1 & 4673.4924 & -0.0054 \\
\hline 6 & 2 & 5 & 6 & 1 & 6 & 6295.8048 & 0.0105 \\
\hline 6 & 0 & 6 & 6 & 0 & 6 & 7068.4150 & 0.0004 \\
\hline 6 & 1 & 6 & 5 & 1 & 5 & 7440.8881 & -0.0003 \\
\hline 6 & 1 & 5 & 5 & 0 & 5 & 6460.7060 & -0.0033 \\
\hline 6 & 2 & 4 & 5 & 2 & 4 & 3549.9623 & 0.0101 \\
\hline 6 & 2 & 5 & 5 & 3 & 3 & 9935.0778 & 0.0000 \\
\hline 6 & 3 & 4 & 5 & 1 & 4 & 13405.5482 & 0.0009 \\
\hline 6 & 1 & 5 & 5 & 2 & 3 & 4805.2867 & -0.0003 \\
\hline 6 & 2 & 4 & 6 & 0 & 6 & 3289.7290 & 0.0001 \\
\hline 6 & 2 & 5 & 6 & 1 & 5 & 6164.0043 & -0.0006 \\
\hline 6 & 3 & 3 & 6 & 1 & 6 & 4916.4701 & -0.0007 \\
\hline 6 & 3 & 4 & 6 & 2 & 4 & 6467.1809 & -0.0016 \\
\hline 6 & 4 & 2 & 6 & 2 & 5 & 7996.1786 & -0.0059 \\
\hline 6 & 4 & 3 & 6 & 3 & 3 & 8233.7745 & -0.0039 \\
\hline 7 & 1 & 7 & 6 & 3 & 4 & 8339.9758 & 0.0014 \\
\hline 7 & 0 & 7 & 6 & 1 & 6 & 8403.2636 & 0.0013 \\
\hline 7 & 1 & 6 & 6 & 0 & 6 & 9651.8065 & 0.0000 \\
\hline 7 & 2 & 5 & 6 & 1 & 5 & 10219.3937 & 0.0007 \\
\hline 7 & 2 & 6 & 6 & 2 & 4 & 9195.9848 & -0.0026 \\
\hline 7 & 3 & 4 & 6 & 2 & 5 & 9909.8961 & 0.0010 \\
\hline 7 & 3 & 5 & 6 & 3 & 3 & 9581.9395 & -0.0007 \\
\hline 7 & 4 & 3 & 6 & 3 & 4 & 9644.3438 & -0.0007 \\
\hline 7 & 4 & 4 & 6 & 4 & 2 & 9616.1677 & 0.0003 \\
\hline 7 & 5 & 2 & 6 & 4 & 3 & 9579.2208 & -0.0016 \\
\hline 7 & 5 & 3 & 6 & 5 & 1 & 9578.2891 & 0.0002 \\
\hline 7 & 6 & 1 & 6 & 5 & 2 & 9552.4681 & 0.0024 \\
\hline 7 & 6 & 2 & 6 & 6 & 0 & 9552.4681 & 0.0024 \\
\hline 7 & 1 & 6 & 6 & 6 & 1 & 5985.3447 & 0.0147 \\
\hline 7 & 2 & 6 & 7 & 1 & 7 & 7088.5218 & 0.0024 \\
\hline 7 & 3 & 5 & 7 & 0 & 7 & 7887.8341 & 0.0107 \\
\hline 7 & 0 & 7 & 7 & 1 & 6 & 8271.4734 & 0.0004 \\
\hline 7 & 1 & 7 & 6 & 1 & 6 & 8471.7631 & -0.0005 \\
\hline 7 & 1 & 6 & 6 & 2 & 5 & 8161.2943 & -0.0050 \\
\hline
\end{tabular}


Table S5 (Continued).

\begin{tabular}{|c|c|c|c|c|c|c|c|}
\hline $\mathbf{J}^{\prime}$ & $\mathrm{K}_{-1}^{\prime}$ & $\mathbf{K}_{+1}^{\prime}$ & $\mathbf{J}^{\prime \prime}$ & $\mathbf{K}_{-1}{ }^{\prime \prime}$ & $\mathbf{K}_{+1}{ }^{\prime \prime}$ & Obs. & Obs.-Cal. \\
\hline 7 & 2 & 5 & 6 & 3 & 4 & 5551.4345 & 0.0023 \\
\hline 7 & 2 & 6 & 6 & 1 & 5 & 10686.4961 & 0.0014 \\
\hline 7 & 1 & 6 & 7 & 0 & 7 & 6053.8322 & 0.0008 \\
\hline 7 & 2 & 5 & 7 & 1 & 6 & 3857.3149 & -0.0003 \\
\hline 7 & 2 & 6 & 7 & 1 & 7 & 7020.0166 & -0.0013 \\
\hline 7 & 3 & 4 & 7 & 2 & 5 & 4606.9730 & 0.0000 \\
\hline 7 & 3 & 5 & 7 & 2 & 6 & 6853.1359 & 0.0006 \\
\hline 7 & 4 & 3 & 7 & 3 & 4 & 7730.6333 & -0.0007 \\
\hline 7 & 4 & 4 & 7 & 3 & 5 & 8267.9969 & -0.0086 \\
\hline 8 & 1 & 8 & 7 & 1 & 7 & 9469.8222 & -0.0023 \\
\hline 8 & 0 & 8 & 7 & 0 & 7 & 9504.0205 & 0.0005 \\
\hline 8 & 1 & 7 & 7 & 1 & 6 & 10782.1764 & 0.0008 \\
\hline 8 & 2 & 6 & 7 & 2 & 5 & 11638.4395 & 0.0045 \\
\hline 8 & 2 & 7 & 7 & 2 & 6 & 10410.9664 & 0.0000 \\
\hline 8 & 3 & 5 & 7 & 3 & 4 & 11485.0411 & 0.0008 \\
\hline 8 & 3 & 6 & 7 & 3 & 5 & 10925.9578 & 0.0011 \\
\hline 8 & 4 & 4 & 7 & 4 & 3 & 11092.8299 & 0.0001 \\
\hline 8 & 4 & 5 & 7 & 4 & 4 & 11018.8622 & -0.0007 \\
\hline 8 & 5 & 3 & 7 & 5 & 2 & 10978.5777 & 0.0002 \\
\hline 8 & 5 & 4 & 7 & 5 & 3 & 10974.9120 & 0.0011 \\
\hline 8 & 7 & 1 & 7 & 7 & 0 & 10913.9908 & -0.0012 \\
\hline 8 & 7 & 2 & 7 & 7 & 1 & 10913.9908 & -0.0012 \\
\hline 8 & 1 & 7 & 8 & 1 & 8 & 7297.6699 & -0.0111 \\
\hline 8 & 0 & 8 & 7 & 1 & 7 & 9435.5179 & -0.0006 \\
\hline 8 & 1 & 8 & 7 & 0 & 7 & 9538.3262 & 0.0002 \\
\hline 8 & 1 & 7 & 7 & 2 & 6 & 9747.4870 & -0.0005 \\
\hline 8 & 2 & 6 & 7 & 3 & 5 & 7607.9160 & -0.0108 \\
\hline 8 & 2 & 7 & 7 & 1 & 6 & 11445.6565 & 0.0020 \\
\hline 8 & 3 & 5 & 7 & 4 & 4 & 3793.5078 & 0.0081 \\
\hline 8 & 1 & 7 & 8 & 0 & 8 & 7331.9879 & 0.0009 \\
\hline 8 & 2 & 6 & 8 & 1 & 7 & 4713.5750 & 0.0003 \\
\hline 8 & 2 & 7 & 8 & 1 & 8 & 7961.1642 & 0.0042 \\
\hline 8 & 3 & 5 & 8 & 2 & 6 & 4453.5778 & -0.0003 \\
\hline 8 & 3 & 6 & 8 & 2 & 7 & 7368.1262 & 0.0006 \\
\hline 8 & 4 & 4 & 8 & 3 & 5 & 7338.4208 & -0.0027 \\
\hline 8 & 4 & 5 & 8 & 3 & 6 & 8360.9072 & -0.0045 \\
\hline 9 & 1 & 9 & 8 & 1 & 8 & 10593.4454 & 0.0020 \\
\hline 9 & 0 & 9 & 8 & 0 & 8 & 10611.0354 & 0.0015 \\
\hline
\end{tabular}


Table S5 (Continued).

\begin{tabular}{|c|c|c|c|c|c|c|c|}
\hline $\mathbf{J}^{\prime}$ & $\mathrm{K}_{-1}^{\prime}$ & $\mathbf{K}_{+1}^{\prime}$ & $\mathbf{J}^{\prime \prime}$ & $\mathbf{K}_{-1}{ }^{\prime \prime}$ & $\mathbf{K}_{+1}{ }^{\prime \prime}$ & Obs. & Obs.-Cal. \\
\hline 9 & 1 & 8 & 8 & 1 & 7 & 11865.2612 & 0.0007 \\
\hline 9 & 2 & 7 & 8 & 2 & 6 & 12978.0765 & -0.0127 \\
\hline 9 & 2 & 8 & 8 & 2 & 7 & 11598.3928 & -0.0009 \\
\hline 9 & 3 & 6 & 8 & 3 & 5 & 13069.0351 & -0.0024 \\
\hline 9 & 3 & 7 & 8 & 3 & 6 & 12242.9804 & 0.0017 \\
\hline 9 & 4 & 5 & 8 & 4 & 4 & 12586.2203 & 0.0024 \\
\hline 9 & 4 & 6 & 8 & 4 & 5 & 12421.1269 & 0.0011 \\
\hline 9 & 5 & 4 & 8 & 5 & 3 & 12393.0700 & 0.0058 \\
\hline 9 & 5 & 5 & 8 & 5 & 4 & 12381.4731 & 0.0065 \\
\hline 9 & 6 & 3 & 8 & 6 & 2 & 12330.1288 & -0.0025 \\
\hline 9 & 6 & 4 & 8 & 6 & 3 & 12329.7329 & 0.0018 \\
\hline 9 & 7 & 2 & 8 & 7 & 1 & 12296.8094 & -0.0015 \\
\hline 9 & 7 & 3 & 8 & 7 & 2 & 12296.8094 & -0.0015 \\
\hline 9 & 8 & 1 & 8 & 8 & 0 & 12275.7408 & -0.0019 \\
\hline 9 & 8 & 2 & 8 & 8 & 1 & 12275.7408 & -0.0019 \\
\hline 9 & 2 & 7 & 9 & 2 & 8 & 5429.7868 & -0.0044 \\
\hline 9 & 0 & 9 & 8 & 1 & 8 & 10576.7294 & 0.0015 \\
\hline 9 & 1 & 9 & 8 & 0 & 8 & 10627.7494 & 0.0000 \\
\hline 9 & 1 & 8 & 8 & 2 & 7 & 11201.7823 & 0.0007 \\
\hline 9 & 2 & 7 & 8 & 3 & 6 & 9660.0585 & -0.0009 \\
\hline 9 & 2 & 8 & 8 & 1 & 7 & 12261.8736 & 0.0009 \\
\hline 9 & 3 & 7 & 8 & 4 & 4 & 3769.0014 & -0.0052 \\
\hline 9 & 1 & 8 & 9 & 0 & 9 & 8586.2149 & 0.0012 \\
\hline 9 & 2 & 7 & 9 & 1 & 8 & 5826.3978 & -0.0056 \\
\hline 9 & 2 & 8 & 9 & 1 & 9 & 8966.0989 & -0.0114 \\
\hline 9 & 3 & 6 & 9 & 2 & 7 & 4544.5260 & -0.0004 \\
\hline 9 & 3 & 7 & 9 & 2 & 8 & 8012.7063 & -0.0041 \\
\hline 9 & 4 & 5 & 9 & 3 & 6 & 6855.6014 & -0.0024 \\
\hline 9 & 4 & 6 & 9 & 3 & 7 & 8539.0436 & -0.0152 \\
\hline 9 & 3 & 7 & 10 & 0 & 10 & 5273.3487 & 0.0078 \\
\hline 9 & 8 & 2 & 10 & 7 & 3 & 3972.6679 & -0.0001 \\
\hline 9 & 9 & 0 & 10 & 8 & 3 & 6382.7106 & 0.0095 \\
\hline 9 & 9 & 1 & 10 & 8 & 2 & 6382.7106 & 0.0095 \\
\hline 10 & 1 & 10 & 9 & 1 & 9 & 11713.4561 & 0.0013 \\
\hline 10 & 0 & 10 & 9 & 0 & 9 & 11722.1897 & -0.0056 \\
\hline 10 & 1 & 9 & 9 & 1 & 8 & 12935.0760 & 0.0031 \\
\hline 10 & 2 & 8 & 9 & 4 & 5 & 2825.3116 & 0.0105 \\
\hline 10 & 2 & 9 & 9 & 2 & 8 & 12762.5935 & 0.0049 \\
\hline
\end{tabular}


Table S5 (Continued).

\begin{tabular}{|c|c|c|c|c|c|c|c|}
\hline $\mathbf{J}^{\prime}$ & $\mathrm{K}_{-1}^{\prime}$ & $\mathbf{K}_{+1}^{\prime}$ & $\mathrm{J}^{\prime \prime}$ & $\mathbf{K}_{-1}{ }^{\prime \prime}$ & $\mathbf{K}_{+1}^{\prime \prime}$ & Obs. & Obs.-Cal. \\
\hline 10 & 2 & 8 & 10 & 2 & 9 & 6892.6242 & -0.0098 \\
\hline 10 & 0 & 10 & 9 & 1 & 9 & 11705.4698 & -0.0101 \\
\hline 10 & 1 & 10 & 9 & 0 & 9 & 11730.1688 & -0.0013 \\
\hline 10 & 1 & 9 & 9 & 2 & 8 & 12538.4616 & 0.0009 \\
\hline 10 & 2 & 8 & 9 & 3 & 7 & 11642.4977 & -0.0145 \\
\hline 10 & 2 & 9 & 9 & 1 & 8 & 13159.1935 & -0.0072 \\
\hline 10 & 3 & 8 & 9 & 4 & 5 & 4711.0491 & 0.0006 \\
\hline 10 & 4 & 6 & 9 & 5 & 5 & 3963.8590 & -0.0168 \\
\hline 10 & 1 & 9 & 10 & 0 & 10 & 9799.0922 & 0.0011 \\
\hline 10 & 2 & 8 & 10 & 1 & 9 & 7116.7605 & -0.0014 \\
\hline 10 & 3 & 7 & 10 & 2 & 8 & 4938.3261 & 0.0000 \\
\hline 10 & 3 & 8 & 10 & 2 & 9 & 8778.3714 & -0.0101 \\
\hline 10 & 4 & 6 & 10 & 3 & 7 & 6371.9084 & -0.0032 \\
\hline 10 & 4 & 7 & 10 & 3 & 8 & 8825.9437 & -0.0090 \\
\hline 10 & 5 & 5 & 10 & 4 & 6 & 9880.8281 & -0.0135 \\
\hline 10 & 0 & 0 & 11 & 9 & 3 & 7381.5132 & -0.0115 \\
\hline 10 & 0 & 1 & 11 & 9 & 2 & 7381.5132 & -0.0115 \\
\hline 11 & 1 & 11 & 10 & 1 & 10 & 12831.5044 & -0.0097 \\
\hline 11 & 0 & 11 & 10 & 0 & 10 & 12835.7450 & -0.0022 \\
\hline 11 & 0 & 11 & 10 & 1 & 10 & 12827.7735 & 0.0011 \\
\hline 11 & 1 & 11 & 10 & 0 & 10 & 12839.4838 & -0.0051 \\
\hline 11 & 2 & 9 & 10 & 3 & 8 & 13494.3961 & -0.0008 \\
\hline 11 & 3 & 8 & 11 & 2 & 9 & 5661.9330 & -0.0030 \\
\hline 11 & 3 & 9 & 10 & 4 & 6 & 5355.2779 & -0.0007 \\
\hline 11 & 2 & 9 & 11 & 1 & 10 & 8485.1747 & 0.0035 \\
\hline 11 & 3 & 9 & 11 & 2 & 10 & 9649.2127 & -0.0029 \\
\hline 11 & 4 & 7 & 11 & 3 & 8 & 6006.4417 & -0.0001 \\
\hline 12 & 3 & 9 & 12 & 3 & 10 & 5880.5035 & -0.0117 \\
\hline 12 & 3 & 10 & 11 & 4 & 7 & 5615.3224 & 0.0047 \\
\hline 12 & 4 & 9 & 11 & 5 & 6 & 5964.1725 & -0.0044 \\
\hline 12 & 2 & 10 & 12 & 1 & 11 & 9848.4743 & 0.0030 \\
\hline 12 & 2 & 11 & 12 & 1 & 12 & 12186.8429 & 0.0060 \\
\hline 12 & 3 & 9 & 12 & 2 & 10 & 6700.3955 & 0.0076 \\
\hline 12 & 4 & 8 & 12 & 3 & 9 & 5872.4731 & -0.0072 \\
\hline 12 & 3 & 10 & 13 & 0 & 13 & 7727.2637 & -0.0014 \\
\hline 13 & 3 & 10 & 13 & 2 & 11 & 7987.6797 & -0.0006 \\
\hline 13 & 4 & 9 & 13 & 3 & 10 & 6053.3235 & 0.0020 \\
\hline 14 & 4 & 10 & 14 & 3 & 11 & 6595.3197 & 0.0031 \\
\hline
\end{tabular}


Table S5 (Continued).

\begin{tabular}{rccccccr}
\hline $\mathbf{J}^{\prime}$ & $\mathbf{K}_{\mathbf{- 1}}{ }^{\prime}$ & $\mathbf{K}_{+\mathbf{1}}{ }^{\prime}$ & $\mathbf{J}^{\prime \prime}$ & $\mathbf{K}_{-\mathbf{1}}{ }^{\prime \prime}$ & $\mathbf{K}_{+\mathbf{1}}{ }^{\prime \prime}$ & Obs. & \multicolumn{1}{c}{ Obs.-Cal. } \\
\hline 14 & 5 & 9 & 14 & 4 & 10 & 7682.9710 & -0.0019 \\
15 & 4 & 11 & 15 & 3 & 12 & 7503.3608 & 0.0173 \\
15 & 5 & 10 & 15 & 4 & 11 & 7317.3656 & -0.0046 \\
16 & 6 & 11 & 15 & 7 & 8 & 7523.7211 & -0.0012 \\
16 & 5 & 11 & 16 & 4 & 12 & 7257.3570 & 0.0093 \\
16 & 4 & 13 & 17 & 1 & 16 & 7388.9385 & -0.0063 \\
16 & 7 & 10 & 17 & 4 & 13 & 6996.9105 & 0.0025 \\
17 & 7 & 10 & 16 & 8 & 9 & 6586.9439 & -0.0091 \\
17 & 5 & 12 & 17 & 4 & 13 & 7575.2037 & -0.0050 \\
\hline
\end{tabular}


Table S6. Observed rotational transitions and residuals (all the values in $\mathrm{MHz}$ ) for the $\mathrm{B} 2$ conformation of the $m$-anisic acid in the ground vibrational state.

\begin{tabular}{|c|c|c|c|c|c|c|c|}
\hline $\mathbf{J}^{\prime}$ & $\mathbf{K}_{-1}^{\prime}$ & $\mathbf{K}_{+1}^{\prime}$ & $\mathrm{J}^{\prime \prime}$ & $\mathbf{K}_{-1}{ }^{\prime \prime}$ & $\mathbf{K}_{+1}^{\prime \prime}$ & Obs. & Obs.-Cal. \\
\hline 2 & 1 & 2 & 1 & 1 & 1 & 2249.1444 & 0.0001 \\
\hline 2 & 0 & 2 & 1 & 0 & 1 & 2382.2294 & 0.0028 \\
\hline 2 & 1 & 1 & 1 & 1 & 0 & 2531.4317 & -0.0099 \\
\hline 2 & 2 & 1 & 2 & 0 & 2 & 7409.5636 & -0.0061 \\
\hline 3 & 1 & 3 & 2 & 1 & 2 & 3368.8109 & -0.0001 \\
\hline 3 & 0 & 3 & 2 & 0 & 2 & 3553.3460 & -0.0012 \\
\hline 3 & 1 & 2 & 2 & 1 & 1 & 3791.9725 & 0.0041 \\
\hline 3 & 2 & 1 & 2 & 2 & 0 & 3617.5294 & -0.0014 \\
\hline 3 & 2 & 2 & 2 & 2 & 1 & 3585.4427 & 0.0036 \\
\hline 4 & 1 & 4 & 3 & 1 & 3 & 4482.9864 & 0.0003 \\
\hline 4 & 0 & 4 & 3 & 0 & 3 & 4701.4636 & 0.0022 \\
\hline 4 & 1 & 3 & 3 & 1 & 2 & 5045.8562 & 0.0039 \\
\hline 4 & 2 & 2 & 3 & 2 & 1 & 4853.4237 & 0.0029 \\
\hline 4 & 2 & 3 & 3 & 2 & 2 & 4774.3062 & 0.0003 \\
\hline 4 & 3 & 1 & 3 & 3 & 0 & 4797.6095 & -0.0052 \\
\hline 4 & 3 & 2 & 3 & 3 & 1 & 4795.8899 & 0.0036 \\
\hline 4 & 2 & 3 & 4 & 0 & 4 & 7514.5024 & -0.0036 \\
\hline 5 & 1 & 5 & 4 & 1 & 4 & 5590.4692 & 0.0001 \\
\hline 5 & 0 & 5 & 4 & 0 & 4 & 5821.8013 & 0.0020 \\
\hline 5 & 1 & 4 & 4 & 1 & 3 & 6290.1926 & 0.0029 \\
\hline 5 & 2 & 3 & 4 & 2 & 2 & 6111.6732 & 0.0011 \\
\hline 5 & 2 & 4 & 4 & 2 & 3 & 5957.8113 & 0.0015 \\
\hline 5 & 3 & 2 & 4 & 3 & 1 & 6006.5692 & 0.0006 \\
\hline 5 & 3 & 3 & 4 & 3 & 2 & 6000.5496 & 0.0035 \\
\hline 5 & 4 & 1 & 4 & 4 & 0 & 5993.6635 & -0.0212 \\
\hline 5 & 4 & 2 & 4 & 4 & 1 & 5993.6635 & -0.0212 \\
\hline 5 & 2 & 4 & 5 & 0 & 5 & 7650.5254 & 0.0089 \\
\hline 6 & 1 & 6 & 5 & 1 & 5 & 6690.5362 & 0.0005 \\
\hline 6 & 0 & 6 & 5 & 0 & 5 & 6913.6416 & 0.0031 \\
\hline 6 & 1 & 5 & 5 & 1 & 4 & 7521.5010 & 0.0037 \\
\hline 6 & 2 & 4 & 5 & 2 & 3 & 7391.5884 & 0.0004 \\
\hline 6 & 2 & 5 & 5 & 2 & 4 & 7134.6620 & 0.0029 \\
\hline 6 & 3 & 3 & 5 & 3 & 2 & 7223.5020 & 0.0021 \\
\hline 6 & 3 & 4 & 5 & 3 & 3 & 7207.5748 & 0.0031 \\
\hline 6 & 4 & 2 & 5 & 4 & 1 & 7198.5095 & -0.0019 \\
\hline 6 & 4 & 3 & 5 & 4 & 2 & 7198.2076 & 0.0034 \\
\hline 6 & 5 & 1 & 5 & 5 & 0 & 7191.0643 & -0.0073 \\
\hline
\end{tabular}


Table S6 (Continued).

\begin{tabular}{|c|c|c|c|c|c|c|c|}
\hline $\mathbf{J}^{\prime}$ & $\mathbf{K}_{-1}^{\prime}$ & $K_{+1}^{\prime}$ & $\mathrm{J}^{\prime \prime}$ & $\mathbf{K}_{-1}{ }^{\prime \prime}$ & $\mathbf{K}_{+1}{ }^{\prime \prime}$ & Obs. & Obs.-Cal. \\
\hline 6 & 5 & 2 & 5 & 5 & 1 & 7191.0643 & -0.0073 \\
\hline 7 & 1 & 7 & 6 & 1 & 6 & 7782.9666 & -0.0017 \\
\hline 7 & 0 & 7 & 6 & 0 & 6 & 7981.0949 & 0.0005 \\
\hline 7 & 1 & 6 & 6 & 1 & 5 & 8735.6514 & 0.0003 \\
\hline 7 & 2 & 5 & 6 & 2 & 4 & 8687.5368 & -0.0015 \\
\hline 7 & 2 & 6 & 6 & 2 & 5 & 8303.6335 & 0.0012 \\
\hline 7 & 3 & 4 & 6 & 3 & 3 & 8451.5240 & 0.0011 \\
\hline 7 & 3 & 5 & 6 & 3 & 4 & 8416.1676 & 0.0015 \\
\hline 7 & 4 & 3 & 6 & 4 & 2 & 8406.7951 & 0.0037 \\
\hline 7 & 5 & 2 & 6 & 5 & 1 & 8394.6851 & -0.0078 \\
\hline 7 & 5 & 3 & 6 & 5 & 2 & 8394.6851 & -0.0078 \\
\hline 8 & 1 & 8 & 7 & 1 & 7 & 8868.0135 & 0.0002 \\
\hline 8 & 0 & 8 & 7 & 0 & 7 & 9031.7354 & -0.0004 \\
\hline 8 & 1 & 7 & 7 & 1 & 6 & 9927.9854 & -0.0007 \\
\hline 8 & 2 & 6 & 7 & 2 & 5 & 9990.2637 & -0.0017 \\
\hline 8 & 2 & 7 & 7 & 2 & 6 & 9463.6259 & 0.0000 \\
\hline 8 & 3 & 5 & 7 & 3 & 4 & 9694.2539 & -0.0024 \\
\hline 8 & 3 & 6 & 7 & 3 & 5 & 9625.0934 & -0.0004 \\
\hline 8 & 5 & 3 & 7 & 5 & 2 & 9600.6708 & -0.0120 \\
\hline 8 & 5 & 4 & 7 & 5 & 3 & 9600.6708 & -0.0120 \\
\hline 8 & 6 & 2 & 7 & 6 & 1 & 9591.6341 & 0.0017 \\
\hline 8 & 6 & 3 & 7 & 6 & 2 & 9591.6341 & 0.0017 \\
\hline 9 & 1 & 9 & 8 & 1 & 8 & 9946.3146 & -0.0013 \\
\hline 10 & 1 & 9 & 10 & 1 & 10 & 7310.6789 & -0.0006 \\
\hline
\end{tabular}


Table S7. Observed rotational transitions and residuals (all the values in $\mathrm{MHz}$ ) for the $\mathrm{B} 1$ conformation of the $m$-anisic acid in the ground vibrational state.

\begin{tabular}{|c|c|c|c|c|c|c|c|}
\hline $\mathbf{J}^{\prime}$ & $\mathbf{K}_{-1}^{\prime}$ & $\mathbf{K}_{+1}^{\prime}$ & $\mathrm{J}^{\prime \prime}$ & $\mathbf{K}_{-1}{ }^{\prime \prime}$ & $\mathbf{K}_{+1}{ }^{\prime \prime}$ & Obs. & Obs.-Cal. \\
\hline 1 & 1 & 1 & 0 & 0 & 0 & 3033.6064 & 0.0040 \\
\hline 2 & 1 & 2 & 1 & 1 & 1 & 2233.5365 & 0.0002 \\
\hline 2 & 0 & 2 & 1 & 0 & 1 & 2361.6989 & 0.0134 \\
\hline 2 & 1 & 1 & 1 & 1 & 0 & 2504.1393 & -0.0082 \\
\hline 2 & 1 & 2 & 1 & 0 & 1 & 4082.7204 & 0.0028 \\
\hline 2 & 2 & 0 & 1 & 1 & 1 & 8194.1538 & 0.0000 \\
\hline 2 & 2 & 1 & 1 & 1 & 0 & 8051.6909 & -0.0005 \\
\hline 2 & 1 & 1 & 2 & 0 & 2 & 2126.9401 & -0.0090 \\
\hline 2 & 2 & 0 & 2 & 1 & 1 & 5554.7066 & 0.0061 \\
\hline 2 & 2 & 1 & 2 & 1 & 2 & 5953.4558 & -0.0051 \\
\hline 3 & 1 & 3 & 2 & 1 & 2 & 3345.9479 & 0.0042 \\
\hline 3 & 0 & 3 & 2 & 0 & 2 & 3524.7724 & 0.0037 \\
\hline 3 & 1 & 2 & 2 & 1 & 1 & 3751.6224 & -0.0011 \\
\hline 3 & 2 & 1 & 2 & 2 & 0 & 3581.7681 & 0.0119 \\
\hline 3 & 1 & 3 & 2 & 0 & 2 & 5066.9782 & 0.0024 \\
\hline 3 & 2 & 1 & 2 & 1 & 2 & 9542.3654 & -0.0082 \\
\hline 3 & 2 & 2 & 2 & 1 & 1 & 9100.8196 & 0.0133 \\
\hline 3 & 1 & 2 & 3 & 0 & 3 & 2353.8111 & 0.0070 \\
\hline 3 & 2 & 1 & 3 & 1 & 2 & 5384.8378 & 0.0046 \\
\hline 3 & 2 & 2 & 3 & 1 & 3 & 6160.7827 & 0.0030 \\
\hline 4 & 1 & 4 & 3 & 1 & 3 & 4453.4462 & 0.0033 \\
\hline 4 & 0 & 4 & 3 & 0 & 3 & 4667.2921 & -0.0012 \\
\hline 4 & 1 & 3 & 3 & 1 & 2 & 4993.2428 & -0.0029 \\
\hline 4 & 2 & 2 & 3 & 2 & 1 & 4802.4937 & -0.0010 \\
\hline 4 & 2 & 3 & 3 & 2 & 2 & 4732.1110 & -0.0012 \\
\hline 4 & 3 & 1 & 3 & 3 & 0 & 4752.7253 & -0.0044 \\
\hline 4 & 3 & 2 & 3 & 3 & 1 & 4751.3111 & 0.0006 \\
\hline 4 & 1 & 4 & 3 & 0 & 3 & 5995.6505 & 0.0004 \\
\hline 4 & 1 & 3 & 4 & 0 & 4 & 2679.7493 & -0.0071 \\
\hline 4 & 2 & 2 & 4 & 1 & 3 & 5194.0834 & 0.0012 \\
\hline 4 & 2 & 3 & 4 & 1 & 4 & 6439.4509 & 0.0019 \\
\hline 5 & 1 & 5 & 4 & 1 & 4 & 5554.9174 & -0.0010 \\
\hline 5 & 0 & 5 & 4 & 0 & 4 & 5784.6532 & 0.0002 \\
\hline 5 & 1 & 4 & 4 & 1 & 3 & 6226.4999 & 0.0029 \\
\hline 5 & 2 & 3 & 4 & 2 & 2 & 6043.6004 & 0.0020 \\
\hline 5 & 2 & 4 & 4 & 2 & 3 & 5906.2023 & 0.0005 \\
\hline 5 & 3 & 2 & 4 & 3 & 1 & 5949.2482 & -0.0032 \\
\hline
\end{tabular}


Table S7 (Continued).

\begin{tabular}{|c|c|c|c|c|c|c|c|}
\hline $\mathbf{J}^{\prime}$ & $\mathrm{K}_{-1}^{\prime}$ & $\mathbf{K}_{+1}^{\prime}$ & $\mathrm{J}^{\prime \prime}$ & $\mathbf{K}_{-1}{ }^{\prime \prime}$ & $\mathbf{K}_{+1}{ }^{\prime \prime}$ & Obs. & Obs.-Cal. \\
\hline 5 & 3 & 3 & 4 & 3 & 2 & 5944.3097 & 0.0066 \\
\hline 5 & 0 & 5 & 4 & 1 & 4 & 4456.2943 & -0.0018 \\
\hline 5 & 1 & 5 & 4 & 0 & 4 & 6883.2750 & -0.0001 \\
\hline 5 & 1 & 4 & 5 & 0 & 5 & 3121.6026 & 0.0021 \\
\hline 5 & 2 & 3 & 5 & 1 & 4 & 5011.1874 & 0.0038 \\
\hline 5 & 2 & 4 & 5 & 1 & 5 & 6790.7329 & 0.0006 \\
\hline 5 & 3 & 2 & 5 & 2 & 3 & 9409.0187 & 0.0129 \\
\hline 6 & 1 & 6 & 5 & 1 & 5 & 6649.6523 & -0.0008 \\
\hline 6 & 0 & 6 & 5 & 0 & 5 & 6875.4952 & 0.0015 \\
\hline 6 & 1 & 5 & 5 & 1 & 4 & 7448.3801 & 0.0002 \\
\hline 6 & 2 & 4 & 5 & 2 & 3 & 7305.1694 & -0.0015 \\
\hline 6 & 2 & 5 & 5 & 2 & 4 & 7074.3785 & 0.0000 \\
\hline 6 & 3 & 3 & 5 & 3 & 2 & 7152.6604 & 0.0011 \\
\hline 6 & 3 & 4 & 5 & 3 & 3 & 7139.5583 & 0.0024 \\
\hline 6 & 4 & 3 & 5 & 4 & 2 & 7130.7844 & 0.0064 \\
\hline 6 & 5 & 1 & 5 & 5 & 0 & 7124.4221 & -0.0115 \\
\hline 6 & 5 & 2 & 5 & 5 & 1 & 7124.4221 & -0.0115 \\
\hline 6 & 0 & 6 & 5 & 1 & 5 & 5776.8678 & -0.0035 \\
\hline 6 & 1 & 6 & 5 & 0 & 5 & 7748.2746 & -0.0009 \\
\hline 6 & 1 & 5 & 6 & 0 & 6 & 3694.4965 & 0.0097 \\
\hline 6 & 2 & 4 & 6 & 1 & 5 & 4867.9755 & 0.0009 \\
\hline 6 & 2 & 5 & 6 & 1 & 6 & 7215.4641 & 0.0064 \\
\hline 6 & 3 & 3 & 6 & 2 & 4 & 9256.4832 & -0.0108 \\
\hline 7 & 1 & 7 & 6 & 1 & 6 & 7737.3584 & -0.0014 \\
\hline 7 & 0 & 7 & 6 & 0 & 6 & 7942.6214 & 0.0111 \\
\hline 7 & 1 & 6 & 6 & 1 & 5 & 8655.3579 & 0.0089 \\
\hline 7 & 2 & 5 & 6 & 2 & 4 & 8583.0860 & -0.0078 \\
\hline 7 & 2 & 6 & 6 & 2 & 5 & 8235.5387 & -0.0045 \\
\hline 7 & 3 & 4 & 6 & 3 & 3 & 8365.5995 & 0.0018 \\
\hline 7 & 3 & 5 & 6 & 3 & 4 & 8336.4461 & -0.0134 \\
\hline 7 & 0 & 7 & 6 & 1 & 6 & 7069.8278 & -0.0007 \\
\hline 7 & 1 & 7 & 6 & 0 & 6 & 8610.1412 & -0.0004 \\
\hline 7 & 1 & 6 & 6 & 2 & 5 & 4261.5967 & 0.0005 \\
\hline 7 & 1 & 6 & 7 & 0 & 7 & 4407.2331 & 0.0077 \\
\hline 7 & 2 & 5 & 7 & 1 & 6 & 4795.7226 & 0.0031 \\
\hline 7 & 2 & 6 & 7 & 1 & 7 & 7713.6449 & 0.0039 \\
\hline 7 & 3 & 4 & 7 & 2 & 5 & 9038.9840 & -0.0139 \\
\hline 7 & 3 & 5 & 7 & 2 & 6 & 9811.9193 & -0.0047 \\
\hline
\end{tabular}


Table S7 (Continued).

\begin{tabular}{|c|c|c|c|c|c|c|c|}
\hline $\mathbf{J}^{\prime}$ & $\mathrm{K}_{-1}^{\prime}$ & $\mathbf{K}_{+1}^{\prime}$ & $\mathrm{J}^{\prime \prime}$ & $\mathbf{K}_{-1}{ }^{\prime \prime}$ & $\mathbf{K}_{+1}{ }^{\prime \prime}$ & Obs. & Obs.-Cal. \\
\hline 8 & 1 & 8 & 7 & 1 & 7 & 8818.1718 & 0.0067 \\
\hline 8 & 0 & 8 & 7 & 0 & 7 & 8992.2053 & -0.0030 \\
\hline 8 & 1 & 7 & 7 & 1 & 6 & 9843.3581 & -0.0032 \\
\hline 8 & 2 & 6 & 7 & 2 & 5 & 9869.7051 & -0.0040 \\
\hline 8 & 3 & 5 & 7 & 3 & 4 & 9591.2061 & 0.0062 \\
\hline 8 & 3 & 6 & 7 & 3 & 5 & 9533.9851 & -0.0064 \\
\hline 8 & 5 & 3 & 7 & 5 & 2 & 9510.3998 & -0.0037 \\
\hline 8 & 5 & 4 & 7 & 5 & 3 & 9510.3998 & -0.0037 \\
\hline 8 & 6 & 2 & 7 & 6 & 1 & 9502.3876 & 0.0094 \\
\hline 8 & 6 & 3 & 7 & 6 & 2 & 9502.3876 & 0.0094 \\
\hline 8 & 7 & 1 & 7 & 7 & 0 & 9497.6378 & -0.0053 \\
\hline 8 & 7 & 2 & 7 & 7 & 1 & 9497.6378 & -0.0053 \\
\hline 8 & 0 & 8 & 7 & 1 & 7 & 8324.6820 & 0.0049 \\
\hline 8 & 1 & 8 & 7 & 0 & 7 & 9485.6992 & 0.0028 \\
\hline 8 & 1 & 7 & 7 & 2 & 6 & 5869.4097 & -0.0046 \\
\hline 8 & 1 & 7 & 8 & 0 & 8 & 5258.3757 & -0.0026 \\
\hline 8 & 2 & 6 & 8 & 1 & 7 & 4822.0737 & 0.0064 \\
\hline 8 & 3 & 5 & 8 & 2 & 6 & 8760.4819 & -0.0066 \\
\hline 9 & 1 & 9 & 8 & 1 & 8 & 9892.5373 & -0.0083 \\
\hline 9 & 1 & 8 & 9 & 1 & 9 & 5880.5035 & -0.0080 \\
\hline 9 & 0 & 9 & 8 & 1 & 8 & 9538.1706 & 0.0031 \\
\hline 9 & 1 & 8 & 8 & 2 & 7 & 7488.8889 & -0.0051 \\
\hline 9 & 1 & 8 & 9 & 0 & 9 & 6234.8916 & 0.0019 \\
\hline 9 & 2 & 7 & 9 & 1 & 8 & 4969.9002 & 0.0035 \\
\hline 9 & 2 & 8 & 9 & 1 & 9 & 8924.5460 & -0.0104 \\
\hline 10 & 2 & 8 & 9 & 3 & 7 & 4204.5792 & 0.0043 \\
\hline 10 & 1 & 9 & 10 & 0 & 10 & 7313.6899 & 0.0020 \\
\hline 10 & 2 & 8 & 10 & 1 & 9 & 5257.7252 & 0.0020 \\
\hline 11 & 2 & 9 & 10 & 3 & 8 & 5976.1492 & -0.0057 \\
\hline 11 & 2 & 9 & 11 & 1 & 10 & 5700.0350 & -0.0041 \\
\hline 11 & 3 & 8 & 11 & 2 & 9 & 7773.6268 & -0.0030 \\
\hline 12 & 2 & 10 & 11 & 3 & 9 & 7800.9153 & 0.0077 \\
\hline 12 & 2 & 10 & 12 & 1 & 11 & 6306.2233 & -0.0062 \\
\hline 12 & 3 & 9 & 12 & 2 & 10 & 7506.1581 & 0.0046 \\
\hline 13 & 3 & 10 & 13 & 2 & 11 & 7331.1360 & 0.0035 \\
\hline 14 & 2 & 12 & 14 & 1 & 13 & 8007.4423 & 0.0121 \\
\hline 14 & 3 & 11 & 14 & 2 & 12 & 7280.8736 & -0.0031 \\
\hline 14 & 3 & 11 & 14 & 3 & 12 & 2173.5669 & -0.0041 \\
\hline
\end{tabular}


Table S7 (Continued).

\begin{tabular}{rrrrrrrr}
\hline $\mathbf{J}^{\prime}$ & $\mathbf{K}_{-\mathbf{1}}{ }^{\prime}$ & $\mathbf{K}_{+\mathbf{1}}{ }^{\prime}$ & $\mathbf{J}^{\prime \prime}$ & $\mathbf{K}_{-\mathbf{1}}{ }^{\prime \prime}$ & $\mathbf{K}_{+\mathbf{1}}{ }^{\prime \prime}$ & Obs. & Obs.-Cal. \\
\hline 15 & 3 & 12 & 15 & 2 & 13 & 7381.5132 & -0.0016 \\
15 & 2 & 13 & 16 & 1 & 16 & 4994.4105 & -0.0009 \\
16 & 3 & 13 & 15 & 4 & 12 & 9079.6917 & -0.0010 \\
\hline
\end{tabular}


Table S8. Observed rotational transitions and residuals (all the values in $\mathrm{MHz}$ ) for the A1-F1 conformer of the $m$-anisic acid - formic acid complex in the ground vibrational state.

\begin{tabular}{|c|c|c|c|c|c|c|c|}
\hline $\mathbf{J}^{\prime}$ & $\mathrm{K}_{-1}{ }^{\prime}$ & $\mathbf{K}_{+1}^{\prime}$ & $\mathbf{J}^{\prime \prime}$ & $\mathbf{K}_{-1}{ }^{\prime \prime}$ & $\mathbf{K}_{+1}{ }^{\prime \prime}$ & Obs. & Obs.-Cal. \\
\hline 3 & 3 & 1 & 2 & 2 & 0 & 7151.6787 & 0.0124 \\
\hline 4 & 3 & 2 & 3 & 2 & 1 & 7697.3041 & -0.0015 \\
\hline 5 & 2 & 3 & 4 & 1 & 4 & 6386.0057 & 0.0125 \\
\hline 6 & 2 & 5 & 5 & 1 & 4 & 6196.9083 & 0.0083 \\
\hline 7 & 2 & 6 & 6 & 1 & 5 & 6587.1882 & 0.0058 \\
\hline 8 & 2 & 7 & 7 & 1 & 6 & 6953.6232 & 0.0021 \\
\hline 8 & 4 & 4 & 8 & 3 & 5 & 7667.9186 & 0.0019 \\
\hline 9 & 2 & 8 & 8 & 1 & 7 & 7298.4007 & 0.0036 \\
\hline 9 & 4 & 5 & 9 & 3 & 6 & 7643.8977 & 0.0143 \\
\hline 10 & 2 & 9 & 9 & 1 & 8 & 7624.5166 & -0.0006 \\
\hline 10 & 4 & 7 & 10 & 3 & 8 & 7679.7756 & 0.0054 \\
\hline 11 & 1 & 10 & 10 & 1 & 9 & 6208.2771 & -0.0015 \\
\hline 11 & 2 & 9 & 10 & 2 & 8 & 6289.0785 & 0.0035 \\
\hline 11 & 2 & 10 & 10 & 2 & 9 & 5980.4344 & 0.0159 \\
\hline 11 & 3 & 8 & 10 & 3 & 7 & 6132.8004 & -0.0037 \\
\hline 11 & 3 & 9 & 10 & 3 & 8 & 6079.1513 & -0.0060 \\
\hline 11 & 4 & 7 & 10 & 4 & 6 & 6080.8537 & -0.0003 \\
\hline 11 & 4 & 8 & 10 & 4 & 7 & 6077.9806 & -0.0062 \\
\hline 11 & 5 & 6 & 10 & 5 & 5 & 6068.3579 & -0.0137 \\
\hline 11 & 6 & 5 & 10 & 6 & 4 & 6062.5958 & 0.0027 \\
\hline 11 & 6 & 6 & 10 & 6 & 5 & 6062.5958 & 0.0027 \\
\hline 11 & 7 & 4 & 10 & 7 & 3 & 6059.2087 & -0.0016 \\
\hline 11 & 7 & 5 & 10 & 7 & 4 & 6059.2087 & -0.0016 \\
\hline 11 & 4 & 7 & 11 & 3 & 8 & 7555.9148 & 0.0024 \\
\hline 12 & 1 & 12 & 11 & 1 & 11 & 6174.6479 & 0.0030 \\
\hline 12 & 0 & 12 & 11 & 0 & 11 & 6225.4817 & 0.0044 \\
\hline 12 & 1 & 11 & 11 & 1 & 10 & 6738.4583 & -0.0009 \\
\hline 12 & 2 & 10 & 11 & 2 & 9 & 6875.3112 & 0.0069 \\
\hline 12 & 2 & 11 & 11 & 2 & 10 & 6509.9226 & 0.0007 \\
\hline 12 & 3 & 9 & 11 & 3 & 8 & 6713.1475 & 0.0054 \\
\hline 12 & 3 & 10 & 11 & 3 & 9 & 6632.4816 & -0.0093 \\
\hline 12 & 4 & 8 & 11 & 4 & 7 & 6640.9625 & -0.0003 \\
\hline 12 & 4 & 9 & 11 & 4 & 8 & 6635.6312 & -0.0060 \\
\hline 12 & 5 & 7 & 11 & 5 & 6 & 6623.8871 & 0.0041 \\
\hline 12 & 5 & 8 & 11 & 5 & 7 & 6623.7059 & -0.0115 \\
\hline 12 & 6 & 6 & 11 & 6 & 5 & 6616.3159 & -0.0106 \\
\hline 12 & 6 & 7 & 11 & 6 & 6 & 6616.3159 & -0.0106 \\
\hline
\end{tabular}


Table S8 (Continued).

\begin{tabular}{|c|c|c|c|c|c|c|c|}
\hline $\mathbf{J}^{\prime}$ & $\mathrm{K}_{-1}^{\prime}$ & $\mathbf{K}_{+1}^{\prime}$ & $\mathrm{J}^{\prime \prime}$ & $\mathbf{K}_{-1}{ }^{\prime \prime}$ & $\mathbf{K}_{+1}{ }^{\prime \prime}$ & Obs. & Obs.-Cal. \\
\hline 12 & 7 & 5 & 11 & 7 & 4 & 6611.9239 & -0.0064 \\
\hline 12 & 7 & 6 & 11 & 7 & 5 & 6611.9239 & -0.0064 \\
\hline 12 & 8 & 4 & 11 & 8 & 3 & 6609.1118 & -0.0051 \\
\hline 12 & 8 & 5 & 11 & 8 & 4 & 6609.1118 & -0.0051 \\
\hline 12 & 0 & 12 & 11 & 1 & 11 & 6026.1974 & 0.0059 \\
\hline 12 & 1 & 12 & 11 & 0 & 11 & 6373.9278 & -0.0028 \\
\hline 12 & 4 & 9 & 12 & 3 & 10 & 7681.7447 & -0.0011 \\
\hline 13 & 1 & 13 & 12 & 1 & 12 & 6676.6132 & 0.0010 \\
\hline 13 & 0 & 13 & 12 & 0 & 12 & 6715.9942 & 0.0019 \\
\hline 13 & 1 & 12 & 12 & 1 & 11 & 7258.9448 & -0.0077 \\
\hline 13 & 2 & 11 & 12 & 2 & 10 & 7456.6390 & -0.0037 \\
\hline 13 & 2 & 12 & 12 & 2 & 11 & 7036.1310 & 0.0009 \\
\hline 13 & 3 & 10 & 12 & 3 & 9 & 7300.1375 & -0.0018 \\
\hline 13 & 3 & 11 & 12 & 3 & 10 & 7184.4834 & 0.0024 \\
\hline 13 & 4 & 9 & 12 & 4 & 8 & 7203.5690 & 0.0038 \\
\hline 13 & 4 & 10 & 12 & 4 & 9 & 7194.2188 & 0.0055 \\
\hline 13 & 5 & 8 & 12 & 5 & 7 & 7180.4452 & -0.0034 \\
\hline 13 & 6 & 7 & 12 & 6 & 6 & 7170.7301 & -0.0101 \\
\hline 13 & 6 & 8 & 12 & 6 & 7 & 7170.7301 & -0.0101 \\
\hline 13 & 7 & 6 & 12 & 7 & 5 & 7165.1484 & 0.0054 \\
\hline 13 & 7 & 7 & 12 & 7 & 6 & 7165.1484 & 0.0054 \\
\hline 13 & 0 & 13 & 12 & 1 & 12 & 6567.5315 & -0.0073 \\
\hline 13 & 1 & 13 & 12 & 0 & 12 & 6825.0665 & 0.0010 \\
\hline 13 & 3 & 11 & 13 & 2 & 12 & 6115.2053 & 0.0059 \\
\hline 13 & 4 & 9 & 13 & 3 & 10 & 7387.1605 & 0.0015 \\
\hline 13 & 4 & 10 & 13 & 3 & 11 & 7691.4716 & -0.0066 \\
\hline 14 & 1 & 14 & 13 & 1 & 13 & 7177.2322 & -0.0015 \\
\hline 14 & 0 & 14 & 13 & 0 & 13 & 7207.0954 & 0.0034 \\
\hline 14 & 1 & 13 & 13 & 1 & 12 & 7769.7838 & -0.0055 \\
\hline 14 & 2 & 13 & 13 & 2 & 12 & 7559.0021 & 0.0073 \\
\hline 14 & 3 & 11 & 13 & 3 & 10 & 7893.4721 & 0.0037 \\
\hline 14 & 3 & 12 & 13 & 3 & 11 & 7734.6457 & -0.0075 \\
\hline 14 & 4 & 10 & 13 & 4 & 9 & 7769.2130 & 0.0009 \\
\hline 14 & 4 & 11 & 13 & 4 & 10 & 7753.5544 & -0.0091 \\
\hline 14 & 5 & 9 & 13 & 5 & 8 & 7738.1988 & -0.0008 \\
\hline 14 & 5 & 10 & 13 & 5 & 9 & 7737.4998 & -0.0045 \\
\hline 14 & 6 & 8 & 13 & 6 & 7 & 7725.9000 & 0.0058 \\
\hline 14 & 6 & 9 & 13 & 6 & 8 & 7725.9000 & 0.0058 \\
\hline
\end{tabular}


Table S8 (Continued).

\begin{tabular}{rrrrrrrr}
\hline $\mathbf{J}^{\prime}$ & $\mathbf{K}_{-\mathbf{1}}{ }^{\prime}$ & $\mathbf{K}_{+\mathbf{1}}{ }^{\prime}$ & $\mathbf{J}^{\prime \prime}$ & $\mathbf{K}_{-\mathbf{1}}{ }^{\prime \prime}$ & $\mathbf{K}_{+\mathbf{1}}{ }^{\prime \prime}$ & Obs. & Obs.-Cal. \\
\hline 14 & 7 & 7 & 13 & 7 & 6 & 7718.8903 & -0.0005 \\
14 & 7 & 8 & 13 & 7 & 7 & 7718.8903 & -0.0005 \\
14 & 0 & 14 & 13 & 1 & 13 & 7098.0158 & -0.0028 \\
14 & 1 & 14 & 13 & 0 & 13 & 7286.3115 & 0.0043 \\
14 & 1 & 13 & 13 & 2 & 12 & 6493.5038 & 0.0088 \\
14 & 2 & 13 & 14 & 1 & 14 & 6221.4915 & -0.0074 \\
14 & 4 & 10 & 14 & 3 & 11 & 7262.9044 & 0.0017 \\
15 & 1 & 15 & 14 & 1 & 14 & 7676.7482 & 0.0004 \\
15 & 0 & 15 & 14 & 0 & 14 & 7699.0104 & 0.0163 \\
15 & 0 & 15 & 14 & 1 & 14 & 7619.7882 & 0.0092 \\
15 & 1 & 15 & 14 & 0 & 14 & 7755.9632 & 0.0003 \\
15 & 1 & 14 & 14 & 2 & 13 & 7206.2671 & -0.0068 \\
15 & 2 & 14 & 15 & 1 & 15 & 6623.2795 & -0.0096 \\
15 & 4 & 11 & 15 & 3 & 12 & 7109.4575 & -0.0048 \\
15 & 4 & 12 & 15 & 3 & 13 & 7741.2824 & -0.0154 \\
16 & 3 & 14 & 16 & 2 & 15 & 6727.7992 & 0.0009 \\
16 & 4 & 12 & 16 & 3 & 13 & 6927.9798 & -0.0095 \\
17 & 1 & 16 & 17 & 0 & 17 & 6953.4395 & 0.0152 \\
17 & 3 & 15 & 17 & 2 & 16 & 6989.6402 & -0.0102 \\
18 & 4 & 14 & 18 & 3 & 15 & 6501.8255 & -0.0044 \\
\hline
\end{tabular}


Table S9. Observed rotational transitions and residuals (all the values in $\mathrm{MHz}$ ) for the A2-F1 conformer of the $m$-anisic acid - formic acid complex in the ground vibrational state.

\begin{tabular}{|c|c|c|c|c|c|c|c|}
\hline $\mathbf{J}^{\prime}$ & $\mathbf{K}_{-1}^{\prime}$ & $\mathbf{K}_{+1}^{\prime}$ & $\mathbf{J}^{\prime \prime}$ & $\mathbf{K}_{-1}{ }^{\prime \prime}$ & $\mathbf{K}_{+1}^{\prime \prime}$ & Obs. & Obs.-Cal. \\
\hline 3 & 3 & 1 & 2 & 2 & 0 & 7514.2660 & -0.0075 \\
\hline 5 & 2 & 3 & 4 & 1 & 4 & 6543.2386 & -0.0086 \\
\hline 6 & 2 & 5 & 5 & 1 & 4 & 6411.9339 & 0.0084 \\
\hline 7 & 2 & 6 & 6 & 1 & 5 & 6806.2053 & 0.0112 \\
\hline 9 & 3 & 7 & 9 & 2 & 8 & 6023.1356 & 0.0038 \\
\hline 11 & 1 & 10 & 10 & 1 & 9 & 6126.9280 & -0.0061 \\
\hline 11 & 2 & 9 & 10 & 2 & 8 & 6167.6029 & 0.0046 \\
\hline 11 & 3 & 8 & 10 & 3 & 7 & 6023.7998 & -0.0093 \\
\hline 12 & 1 & 12 & 11 & 1 & 11 & 6120.0111 & -0.0022 \\
\hline 12 & 0 & 12 & 11 & 0 & 11 & 6181.5801 & -0.0107 \\
\hline 12 & 2 & 10 & 11 & 2 & 9 & 6745.4385 & -0.0036 \\
\hline 12 & 2 & 11 & 11 & 2 & 10 & 6430.6118 & 0.0034 \\
\hline 12 & 3 & 10 & 11 & 3 & 9 & 6531.9384 & -0.0055 \\
\hline 12 & 3 & 9 & 11 & 3 & 8 & 6588.8323 & -0.0006 \\
\hline 12 & 4 & 8 & 11 & 4 & 7 & 6534.5372 & -0.0081 \\
\hline 12 & 4 & 9 & 11 & 4 & 8 & 6531.3749 & -0.0032 \\
\hline 12 & 1 & 12 & 11 & 0 & 11 & 6388.7077 & 0.0142 \\
\hline 13 & 1 & 13 & 12 & 1 & 12 & 6618.9291 & -0.0089 \\
\hline 13 & 0 & 13 & 12 & 0 & 12 & 6668.5953 & 0.0006 \\
\hline 13 & 1 & 12 & 12 & 1 & 11 & 7180.1532 & 0.0065 \\
\hline 13 & 2 & 11 & 12 & 2 & 10 & 7320.3107 & 0.0006 \\
\hline 13 & 2 & 12 & 12 & 2 & 11 & 6953.2765 & 0.0079 \\
\hline 13 & 3 & 10 & 12 & 3 & 9 & 7159.3650 & 0.0069 \\
\hline 13 & 3 & 11 & 12 & 3 & 10 & 7076.7784 & 0.0147 \\
\hline 13 & 4 & 9 & 12 & 4 & 8 & 7085.9823 & 0.0042 \\
\hline 13 & 4 & 10 & 12 & 4 & 9 & 7080.3998 & 0.0018 \\
\hline 13 & 5 & 8 & 12 & 5 & 7 & 7068.6157 & 0.0003 \\
\hline 13 & 6 & 7 & 12 & 6 & 6 & 7060.9649 & -0.0082 \\
\hline 13 & 6 & 8 & 12 & 6 & 7 & 7060.9649 & -0.0082 \\
\hline 13 & 7 & 6 & 12 & 7 & 5 & 7056.5465 & 0.0152 \\
\hline 13 & 7 & 7 & 12 & 7 & 6 & 7056.5465 & 0.0152 \\
\hline 13 & 8 & 5 & 12 & 8 & 4 & 7053.6896 & 0.0006 \\
\hline 13 & 8 & 6 & 12 & 8 & 5 & 7053.6896 & 0.0006 \\
\hline 13 & 0 & 13 & 12 & 1 & 12 & 6461.4898 & -0.0023 \\
\hline 14 & 1 & 13 & 13 & 1 & 12 & 7694.4968 & 0.0049 \\
\hline 14 & 0 & 14 & 13 & 0 & 13 & 7155.6613 & -0.0084 \\
\hline 14 & 1 & 14 & 13 & 1 & 13 & 7116.5091 & -0.0064 \\
\hline
\end{tabular}


Table S9 (Continued).

\begin{tabular}{rrrrrrrr}
\hline $\mathrm{J}^{\prime}$ & $\mathrm{K}_{-1}{ }^{\prime}$ & $\mathrm{K}_{+1}{ }^{\prime}$ & $\mathrm{J}^{\prime \prime}$ & $\mathrm{K}_{-1}{ }^{\prime \prime}$ & $\mathrm{K}_{+1}{ }^{\prime \prime}$ & Obs. & \multicolumn{1}{c}{ Obs.-Cal. } \\
\hline 14 & 2 & 12 & 13 & 2 & 11 & 7890.8863 & -0.0085 \\
14 & 2 & 13 & 13 & 2 & 12 & 7473.1061 & 0.0008 \\
14 & 3 & 11 & 13 & 3 & 10 & 7735.5923 & 0.0023 \\
14 & 3 & 12 & 13 & 3 & 11 & 7620.3957 & -0.0074 \\
14 & 4 & 10 & 13 & 4 & 9 & 7639.5761 & -0.0021 \\
14 & 1 & 14 & 13 & 0 & 13 & 7273.9602 & -0.0012 \\
15 & 0 & 15 & 14 & 0 & 14 & 7643.2572 & 0.0094 \\
15 & 1 & 15 & 14 & 1 & 14 & 7612.9361 & -0.0056 \\
15 & 2 & 14 & 14 & 2 & 13 & 7990.0847 & 0.0028 \\
15 & 0 & 15 & 14 & 1 & 14 & 7524.9523 & -0.0037 \\
15 & 1 & 15 & 14 & 0 & 14 & 7731.2373 & 0.0037 \\
15 & 2 & 14 & 15 & 1 & 15 & 6608.6730 & 0.0017 \\
18 & 4 & 14 & 18 & 3 & 15 & 7268.1796 & -0.0039 \\
\hline
\end{tabular}


Table S10. Observed rotational transitions and residuals (all the values in $\mathrm{MHz}$ ) for the B2-F1 conformer of the $m$-anisic acid - formic acid complex in the ground vibrational state.

\begin{tabular}{|c|c|c|c|c|c|c|c|}
\hline $\mathbf{J}^{\prime}$ & $\mathbf{K}_{-1}^{\prime}$ & $\mathbf{K}_{+1}^{\prime}$ & $\mathbf{J}^{\prime \prime}$ & $\mathbf{K}_{-1}{ }^{\prime \prime}$ & $\mathbf{K}_{+1}^{\prime \prime}$ & Obs. & Obs.-Cal. \\
\hline 12 & 1 & 11 & 11 & 1 & 10 & 6123.8696 & -0.0014 \\
\hline 12 & 2 & 10 & 11 & 2 & 9 & 6071.9473 & -0.0006 \\
\hline 12 & 3 & 9 & 11 & 3 & 8 & 5991.3262 & 0.0097 \\
\hline 12 & 3 & 10 & 11 & 3 & 9 & 5982.2998 & 0.0004 \\
\hline 13 & 1 & 13 & 12 & 1 & 12 & 6217.5713 & -0.0017 \\
\hline 13 & 0 & 13 & 12 & 0 & 12 & 6310.4674 & -0.0078 \\
\hline 13 & 1 & 12 & 12 & 1 & 11 & 6625.6515 & -0.0021 \\
\hline 13 & 2 & 11 & 12 & 2 & 10 & 6592.0368 & -0.0059 \\
\hline 13 & 2 & 12 & 12 & 2 & 11 & 6437.4537 & -0.0073 \\
\hline 13 & 3 & 10 & 12 & 3 & 9 & 6495.8122 & -0.0062 \\
\hline 13 & 3 & 11 & 12 & 3 & 10 & 6482.3810 & -0.0025 \\
\hline 13 & 4 & 9 & 12 & 4 & 8 & 6478.6412 & -0.0145 \\
\hline 13 & 4 & 10 & 12 & 4 & 9 & 6478.2494 & -0.0005 \\
\hline 13 & 5 & 8 & 12 & 5 & 7 & 6473.9720 & 0.0074 \\
\hline 13 & 5 & 9 & 12 & 5 & 8 & 6473.9720 & 0.0074 \\
\hline 13 & 6 & 7 & 12 & 6 & 6 & 6471.6372 & 0.0150 \\
\hline 13 & 6 & 8 & 12 & 6 & 7 & 6471.6372 & 0.0150 \\
\hline 14 & 1 & 14 & 13 & 1 & 13 & 6690.4804 & 0.0044 \\
\hline 14 & 0 & 14 & 13 & 0 & 13 & 6776.4293 & -0.0044 \\
\hline 14 & 1 & 13 & 13 & 1 & 12 & 7125.0408 & 0.0052 \\
\hline 14 & 2 & 12 & 13 & 2 & 11 & 7113.3089 & -0.0006 \\
\hline 14 & 2 & 13 & 13 & 2 & 12 & 6927.6330 & 0.0025 \\
\hline 14 & 3 & 11 & 13 & 3 & 10 & 7001.9220 & -0.0018 \\
\hline 14 & 3 & 12 & 13 & 3 & 11 & 6982.5440 & -0.0051 \\
\hline 14 & 4 & 10 & 13 & 4 & 9 & 6979.2092 & 0.0072 \\
\hline 14 & 4 & 11 & 13 & 4 & 10 & 6978.5150 & 0.0012 \\
\hline 14 & 5 & 9 & 13 & 5 & 8 & 6973.2583 & 0.0115 \\
\hline 14 & 6 & 8 & 13 & 6 & 7 & 6970.3275 & 0.0144 \\
\hline 14 & 6 & 9 & 13 & 6 & 8 & 6970.3275 & 0.0144 \\
\hline 14 & 7 & 7 & 13 & 7 & 6 & 6968.5858 & 0.0001 \\
\hline 14 & 7 & 8 & 13 & 7 & 7 & 6968.5858 & 0.0001 \\
\hline 15 & 1 & 15 & 14 & 1 & 14 & 7162.5254 & -0.0032 \\
\hline 15 & 0 & 15 & 14 & 0 & 14 & 7240.6179 & 0.0039 \\
\hline 15 & 1 & 14 & 14 & 1 & 13 & 7621.7238 & -0.0006 \\
\hline 15 & 2 & 13 & 14 & 2 & 12 & 7635.1252 & -0.0001 \\
\hline 15 & 2 & 14 & 14 & 2 & 13 & 7416.7252 & 0.0012 \\
\hline 15 & 3 & 12 & 14 & 3 & 11 & 7509.8567 & -0.0046 \\
\hline
\end{tabular}


Table S10 (Continued).

\begin{tabular}{rrrrrrrr}
\hline $\mathbf{J}^{\prime}$ & $\mathbf{K}_{\mathbf{- 1}}{ }^{\prime}$ & $\mathbf{K}_{+\mathbf{1}}{ }^{\prime}$ & $\mathbf{J}^{\prime \prime}$ & $\mathbf{K}_{-\mathbf{1}}{ }^{\prime \prime}$ & $\mathbf{K}_{+\mathbf{1}}{ }^{\prime \prime}$ & Obs. & Obs.-Cal. \\
\hline 15 & 3 & 13 & 14 & 3 & 12 & 7482.7086 & -0.0004 \\
15 & 4 & 12 & 14 & 4 & 11 & 7479.1551 & -0.0125 \\
15 & 5 & 10 & 14 & 5 & 9 & 7472.7975 & -0.0050 \\
15 & 5 & 11 & 14 & 5 & 10 & 7472.7975 & -0.0050 \\
16 & 1 & 16 & 15 & 1 & 15 & 7633.7701 & 0.0017 \\
16 & 0 & 16 & 15 & 0 & 15 & 7703.5256 & 0.0010 \\
16 & 2 & 15 & 15 & 2 & 14 & 7904.6790 & -0.0063 \\
16 & 3 & 14 & 15 & 3 & 13 & 7982.7752 & 0.0097 \\
16 & 4 & 13 & 15 & 4 & 12 & 7980.2182 & -0.0007 \\
\hline
\end{tabular}


Table S11. Observed rotational transitions and residuals (all the values in MHz) for the B1-F1 conformer of the $m$-anisic acid - formic acid complex in the ground vibrational state.

\begin{tabular}{|c|c|c|c|c|c|c|c|}
\hline $\mathbf{J}^{\prime}$ & $\mathbf{K}_{-1}^{\prime}$ & $\mathbf{K}_{+1}^{\prime}$ & $\mathbf{J}^{\prime \prime}$ & $\mathbf{K}_{-1}{ }^{\prime \prime}$ & $\mathbf{K}_{+1}^{\prime \prime}$ & Obs. & Obs.-Cal. \\
\hline 12 & 1 & 11 & 11 & 1 & 10 & 6198.2294 & -0.0095 \\
\hline 12 & 2 & 10 & 11 & 2 & 9 & 6164.4140 & -0.0049 \\
\hline 12 & 2 & 11 & 11 & 2 & 10 & 6008.9565 & 0.0078 \\
\hline 12 & 3 & 9 & 11 & 3 & 8 & 6067.6016 & 0.0102 \\
\hline 12 & 3 & 10 & 11 & 3 & 9 & 6054.1225 & -0.0009 \\
\hline 12 & 4 & 8 & 11 & 4 & 7 & 6050.3410 & 0.0028 \\
\hline 12 & 4 & 9 & 11 & 4 & 8 & 6049.9295 & -0.0054 \\
\hline 12 & 5 & 7 & 11 & 5 & 6 & 6045.6267 & 0.0094 \\
\hline 12 & 5 & 8 & 11 & 5 & 7 & 6045.6267 & 0.0094 \\
\hline 12 & 6 & 6 & 11 & 6 & 5 & 6043.2537 & -0.0050 \\
\hline 12 & 6 & 7 & 11 & 6 & 6 & 6043.2537 & -0.0050 \\
\hline 13 & 1 & 13 & 12 & 1 & 12 & 6263.9648 & 0.0026 \\
\hline 13 & 0 & 13 & 12 & 0 & 12 & 6349.7634 & -0.0063 \\
\hline 13 & 1 & 12 & 12 & 1 & 11 & 6703.4264 & -0.0026 \\
\hline 13 & 2 & 11 & 12 & 2 & 10 & 6693.6320 & 0.0049 \\
\hline 13 & 2 & 12 & 12 & 2 & 11 & 6504.1777 & 0.0066 \\
\hline 13 & 3 & 10 & 12 & 3 & 9 & 6580.3168 & 0.0049 \\
\hline 13 & 3 & 11 & 12 & 3 & 10 & 6560.3017 & 0.0026 \\
\hline 13 & 4 & 9 & 12 & 4 & 8 & 6556.9378 & -0.0063 \\
\hline 13 & 4 & 10 & 12 & 4 & 9 & 6556.2282 & -0.0010 \\
\hline 13 & 5 & 8 & 12 & 5 & 7 & 6550.8375 & 0.0093 \\
\hline 13 & 5 & 9 & 12 & 5 & 8 & 6550.8375 & 0.0093 \\
\hline 13 & 6 & 7 & 12 & 6 & 6 & 6547.8231 & -0.0040 \\
\hline 13 & 6 & 8 & 12 & 6 & 7 & 6547.8231 & -0.0040 \\
\hline 14 & 1 & 14 & 13 & 1 & 13 & 6739.3530 & -0.0038 \\
\hline 14 & 0 & 14 & 13 & 0 & 13 & 6816.4918 & -0.0015 \\
\hline 14 & 1 & 13 & 13 & 1 & 12 & 7205.4065 & -0.0027 \\
\hline 14 & 2 & 12 & 13 & 2 & 11 & 7223.4214 & 0.0009 \\
\hline 14 & 2 & 13 & 13 & 2 & 12 & 6998.1159 & -0.0005 \\
\hline 14 & 3 & 11 & 13 & 3 & 10 & 7095.2164 & -0.0032 \\
\hline 14 & 3 & 12 & 13 & 3 & 11 & 7066.4513 & -0.0081 \\
\hline 14 & 4 & 10 & 13 & 4 & 9 & 7064.2005 & 0.0029 \\
\hline 14 & 4 & 11 & 13 & 4 & 10 & 7062.9945 & 0.0080 \\
\hline 14 & 5 & 9 & 13 & 5 & 8 & 7056.3829 & 0.0036 \\
\hline 14 & 5 & 10 & 13 & 5 & 9 & 7056.3829 & 0.0036 \\
\hline 14 & 6 & 8 & 13 & 6 & 7 & 7052.6150 & -0.0125 \\
\hline 14 & 6 & 9 & 13 & 6 & 8 & 7052.6150 & -0.0125 \\
\hline
\end{tabular}


Table S11 (Continued).

\begin{tabular}{rrrrrrrr}
\hline $\mathbf{J}^{\prime}$ & $\mathbf{K}_{\mathbf{- 1}}{ }^{\prime}$ & $\mathbf{K}_{+\mathbf{1}}{ }^{\prime}$ & $\mathbf{J}^{\prime \prime}$ & $\mathbf{K}_{-\mathbf{1}}{ }^{\prime \prime}$ & $\mathbf{K}_{+\mathbf{1}}{ }^{\prime \prime}$ & Obs. & \multicolumn{1}{c}{ Obs.-Cal. } \\
\hline 15 & 1 & 15 & 14 & 1 & 14 & 7213.7907 & -0.0088 \\
15 & 0 & 15 & 14 & 0 & 14 & 7281.8136 & 0.0003 \\
15 & 1 & 14 & 14 & 1 & 13 & 7703.8089 & 0.0007 \\
15 & 2 & 13 & 14 & 2 & 12 & 7752.9929 & -0.0098 \\
15 & 2 & 14 & 14 & 2 & 13 & 7490.7213 & 0.0073 \\
15 & 3 & 12 & 14 & 3 & 11 & 7612.6043 & -0.0002 \\
15 & 3 & 13 & 14 & 3 & 12 & 7572.4718 & -0.0059 \\
15 & 4 & 12 & 14 & 4 & 11 & 7570.2221 & 0.0078 \\
16 & 1 & 16 & 15 & 1 & 15 & 7687.3444 & -0.0070 \\
16 & 0 & 16 & 15 & 0 & 15 & 7746.3047 & 0.0069 \\
16 & 2 & 15 & 15 & 2 & 14 & 7981.9136 & 0.0109 \\
\hline
\end{tabular}

